\title{
15. X-RAY MINERALOGY STUDIES, LEG 7
}

\author{
H. E. Cook, R. W. Rex, W. A. Eklund and B. Murray \\ University of California, Riverside, California
}

\section{INTRODUCTION}

The methods used in the X-ray mineralogy studies have been described briefly in the reports for Legs 1 and 2 and are discussed at greater length in Appendix III of Volume IV. The results of X-ray studies on core samples collected on Leg 7 are presented below.

\section{RESULTS}

\section{Site 61}

This site is located near the western edge of the Marianas Basin, directly east of the Marianas Trench. Site 61 is 35 miles northwest of Site 59 from Leg 6, and was chosen with the objective of reaching the basement in this area, an objective that was not accomplished at Site 59. As the soft upper sediments in this area were cored at Site 59, no cores were taken above 72 meters at Site 61 .

We received material from one Upper Cretaceous core taken from 83 to 89 meters below the mudline in Hole 61.1. Bulk X-ray analyses show primarily highly crystalline cristobalite, with lesser amounts of quartz and plagioclase (Figure 1). These minerals are also present in similar proportions in the $<2$-micron fraction. Montmorillonite is also present in the $<2$-micron fraction (Figure 2). The high cristobalite content of this sample suggests we received primarily the radiolarian chert which was identified in this core and described in the Shipboard Report. No zeolites were detected, although this core was described as having abundant zeolitic clay in the Shipboard Report.

\section{Site 62}

Site 62 is located on the crest of the Euaripik Ridge and was chosen with the objective of recovering basement material from a region which is a maximum distance from the East Pacific Rise and also still on the ocean side of the Andesite Line.

The bulk samples show virtually all calcite, with the exception of a few percentage of quartz, plagioclase, kaolinite and mica which were detected in the uppermost few meters of sediment (Figures 3 and 5). This high calcite content reflects the high percentage of calcareous nannofossils and foraminifera.
The calcite-free, $<2$-micron composited samples show a relatively constant proportion of quartz, plagioclase and kaolinite throughout Hole 62.1 (Figures 4 and 6). These cores range from Middle Miocene to Quaternary.

Montmorillonite is abundant in the $<2$-micron fraction of almost all samples from Hole 62.1. Pyrite is present in small amounts in the $<2$-micron fraction in many cores.

\section{Site 63}

Site 63, which is located in the southeast part of the East Caroline Basin, was chosen for the purpose of determining whether the basement in this region is older than the basalt basement at Site 63, and to determine the nature and age of the upper strong seismic reflectors.

The bulk composited samples from the upper 100 meters contain the highest percentage of kaolinite, montmorillonite, mica, quartz and plagioclase found at this site (Figures 7,9 and 11). This stratigraphic interval which is Late Miocene through Pliocene corresponds with the yellow-brown and pelagic clay interval. Calcite increases steadily with depth; below 120 meters, which is near the Middle-Late Miocene boundary, calcite comprises 99 per cent of each sample analyzed.

The calcite-free $<2$-micron fraction of every sample from this site contains abundant montmorillonite (Figures 8, 10 and 12). In addition to montmorillonite, quartz, plagioclase, kaolinite and mica are present in relatively constant proportions, especially in the closely cored Pliocene through Early Miocene sequence down to 193 meters. Clinoptilolite was only detected in the Late Oligocene, Core 6. A single occurrence of cristobalite was detected a few meters above basement.

\section{Site 64}

Site 64, which is located in the middle of the Ontong-Java Plateau, was chosen in the hope that the thick sediment cover in this area might reveal a history of pelagic sedimentation extending as far back as the Mesozoic. 
The bulk samples consist entirely of calcite which reflects the high calcareous nannofossil content at this site. Minor quartz and plagioclase is also present in Core 1 (Figures 13 and 15).

The $<2$-micron fraction shows the greatest variation in both mineral species and concentrations (Figures 14 and 16). Montmorillonite is generally most abundant. Quartz, plagioclase and kaolinite are present in small concentrations in most cores. Barite occurs in minor amounts from about the 300-meter level in the Middle Miocene to the base of the site at 972 meters in the Middle Eocene. Mica is present in varying concentrations in about half of the cores. Pyrite is also present in minor quantities in a few cores.

\section{Site 65}

Site 65 is located northeast of the Gilbert Islands in the Central Pacific Basin. The region lies within the crestal area of the postulated Darwin Rise, and the site was chosen with the purpose of determining the sedimentary sequence and history of this region.

The composited bulk samples from this site are mainly radiolarian-rich sediments low in calcite (Figures 17 and 19). Cores 2 through 13 range from Pliocene through Oligocene. In addition to the radiolarian amorphous opal, these cores contain quartz and plagioclase, along with montmorillonite in Core 2 and mica in Cores 2, 3 and 5. One occurrence of clinoptilolite was found in Core 12 of Early Miocene age. Cores 14 and 16, which are Oligocene and Late Eocene in age, consist primarily of calcite with minor quartz and plagioclase. These calcite-rich cores are described in the Shipboard Report as of probable turbidite origin. The two Middle Eocene cores underlying the calcareous cores consist of radiolarian oozes and cherts. Core 5 of Hole 65.1, which is Middle Eocene, also contains clinoptilolite.

The composited calcite-free $<2$-micron fraction is also quite amorphous and indicates probable radiolarians, diatoms, and/or volcanic glass (Figures 18 and 20). In the $<2$-micron fraction of Cores 2 through 13 , quartz and plagioclase are present with varying concentrations of kaolinite, mica and montmorillonite. Cores 12, 14 and 16 of Hole 65.0 and Core 2 of Hole 65.1 of Late Eocene and Oligocene age contain clinoptilolite in the $<2$-micron fraction. A single occurrence of siderite was identified in Core 14, Hole 65.0 of Oligocene age.

\section{Site 66}

Site 66 is located in the eastern side of the Central Pacific Basin, west of Christmas Island.

All of the samples from 20 to 126 meters are in the "upper transparent layer" of Oligocene through Pliocene age. These sediments are highly amorphous and correspond to the opaline radiolarian and diatomaceous ooze described in the Shipboard Report. The only calcareous sample in this group is from the Middle Miocene Core 2 of Hole 66.0. Quartz is present in all samples, along with varying amounts of plagioclase, kaolinite, mica and montmorillonite. Barite is present in Core 8 of Hole 66.1, which is Middle Miocene in age. Clinoptilolite is present in Core 3 of this "upper transparent layer" (Figures 21 and 23).

Underlying the siliceous "upper transparent layer" is the "lower transparent layer" of Oligocene to Cretaceous age which consists of stiff, brown pelagic clay underlain by vesicular basalt at 193 meters. The composited bulk samples from this layer consist primarily of quartz, K-feldspar, montmorillonite, mica and palygorskite. The palygorskite occurs from 165 meters below the mudline to the bottom of the hole at 192 meters. This interval ranges in age from Oligocene to Cretaceous. There is no stratigraphic overlap of barite and palygorskite (Figures 22 and 24).

\section{Palygorskite Identification}

The presence of palygorskite in samples from Site 66 was initially suspected on the basis of an XRD peak at $10.5 \AA$. This peak appeared either as a distinct shoulder on the $10.0 \AA$ mica peak or as a separate peak. The peak was not shifted by saturation with glycerol or tri-N-Hexylamine acetate solution. Possible secondary palygorskite peaks were present in the XRD patterns, but interference by other minerals made verification on this basis difficult.

Heating the samples for short periods of time at temperatures up to $400^{\circ} \mathrm{C}$ had no effect on the position or intensity of the $10.5 \AA$ peak. Heating the samples at $450^{\circ} \mathrm{C}$ for one-half hour caused the $10.5 \AA$ peak to irreversibly disappear.

Positive identification of palygorskite in the Site 66 samples by electron microscope methods was difficult because of the abundance of fine-grained mica. The electron micrograph (Plate 1) shows the habit is similar to the palygorskite-rich clays found on Leg 2, Site 12B (Volume II, p. 333). It has been suggested that certain illites strongly resemble palygorskite (Caillere and Henin, 1961). Further study of these samples is planned and findings will be published in a future volume.

\section{Site 67}

Site 67 is located on the Hawaiian Arch about 180 kilometers north of Oahu. The sea floor at this site consists of well-indurated volcanic sandstones, volcanic mudstones, radiolarian ooze and opaline chert. Samples were recovered at only three depths before the drill bit was stopped by impenetrable Eocene chert. 
We received samples from the upper two cores recovered. These are Eocene in age and were taken from 0 to 5 and 23 to 32 meters below mudline. The crystalline, composited bulk material from each core consists mainly of phillipsite with minor quartz and plagioclase (Figures 25 and 27). In the $<2$-micron fractions, phillipsite is abundant in the upper core but is absent in the lower core where montmorillonite is predominant (Figures 26 and 28).

\section{DISCUSSION}

Nodular and bedded cherts were cored and recovered at several sites and at various stratigraphic intervals. A detailed account of their mineralogy and texture is reported elsewhere in this volume.

Barite occurs in Sites 64 and 66. It is stratigraphically most abundant in Site 64, where it occurs through much of the 650-meter thick interval from Middle Miocene to Middle Eocene. In Site 66 it was only recognized in Core 8 of the Middle Miocene. Palygorskite occurs at Site 66 in Early Miocene and older sediments whereas, barite is restricted to the upper 86 meters in the Middle Miocene, a stratigraphic separation of about 80 meters. The absence of any stratigraphic overlap suggests that the two minerals do not have a close genetic tie at least in time. Whether the barite and palygorskite formed in situ authigenically at low temperatures from sea water equilibrium solutions or were formed hydrothermally is not known.

Clinoptilolite was detected at Sites 62, 63, 65 and 66. Its restriction in Site 62 to a stratigraphic interval with volcanic glass suggests a probable authigenic origin with the volcanic glass supplying the necessary cations and silica. There is no obvious genetic correlation regarding clinoptilolite in the other sites. Phillipsite is common in the Early Eocene Core 1 at Site 67. Abundant volcanic constituents including volcanic glass were reported in this stratigraphic interval by the shipboard scientists. This stratigraphic association of phillipsite with volcanic glass also suggests a probable authigenic origin for the phillipsite. Petrographic work would be necessary to confirm or reject this suggested origin for both the phillipsite and clinoptilolite.

\section{REFERENCE}

Caillere, S. and Henin, S., 1961. Palygorskite, In The $X$-ray Identification and Crystal Structures of Clay Minerals. G. Brown (Ed.) London (Mineralogical Society). 


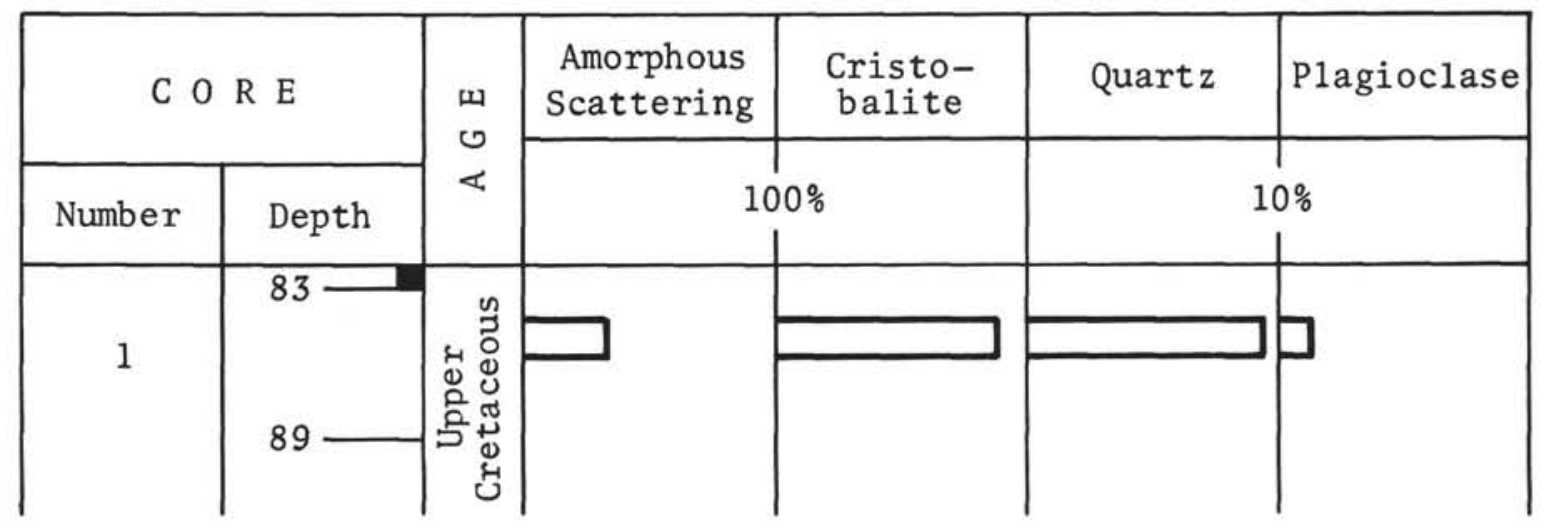

Figure 1. Hole 61A, X-ray results, composited bulk samples.

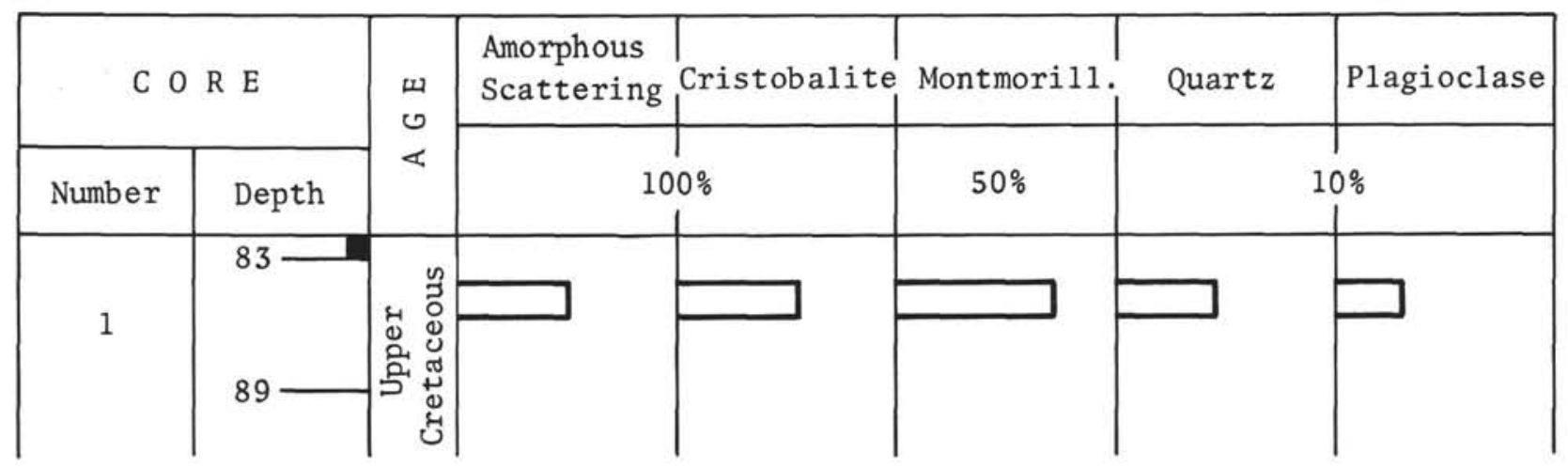

Figure 2. Hole 61A, X-ray results, composited less than $2 \mu$ samples. 


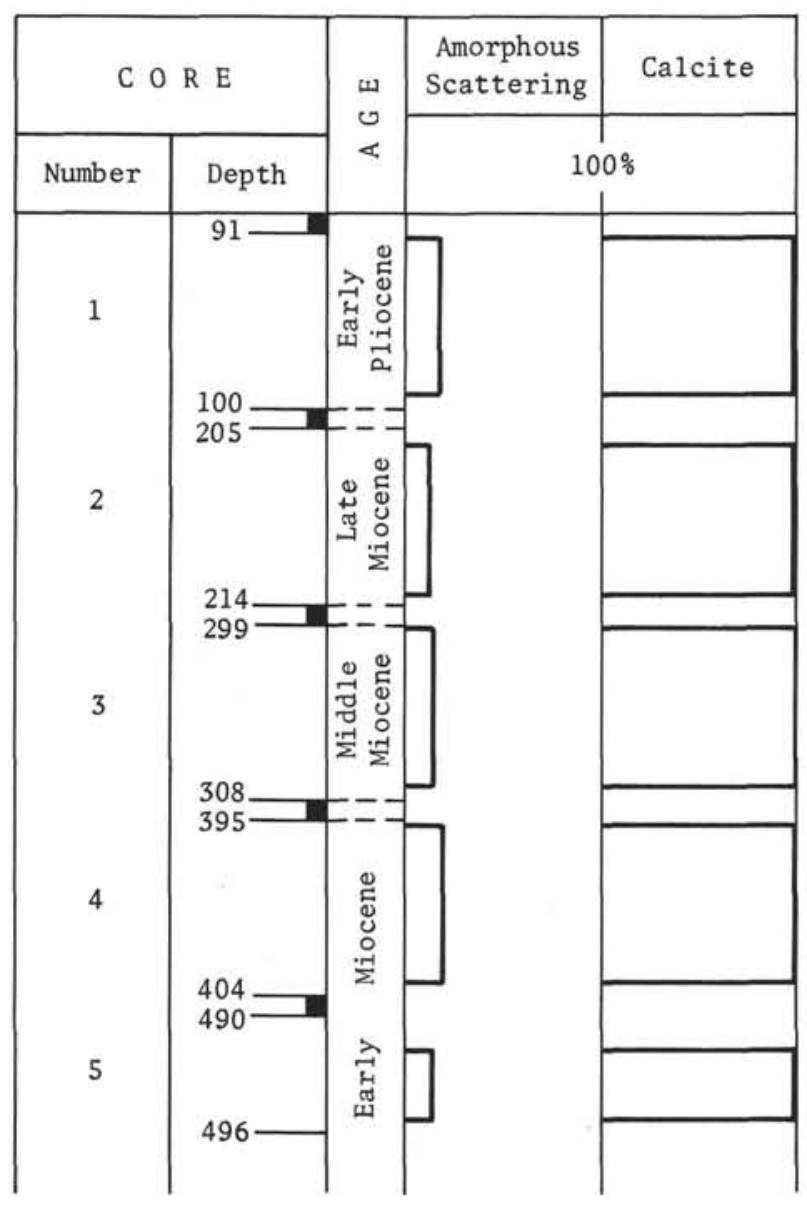

Figure 3. Hole 62, X-ray results, composited bulk samples, 


\begin{tabular}{|c|c|c|c|c|c|c|c|c|c|c|c|}
\hline \multicolumn{2}{|c|}{$C O R E$} & \multirow{2}{*}{$\begin{array}{l}w \\
\vdots \\
<\end{array}$} & \begin{tabular}{|l|l} 
Amorphous \\
Scattering
\end{tabular} & \multirow[t]{2}{*}{ Montmoril11. } & \multirow{2}{*}{$\begin{array}{l}\text { Mica } \\
50 \%\end{array}$} & Quartz & \multirow{2}{*}{\begin{tabular}{|c|} 
Kaol inite \\
$25 \%$
\end{tabular}} & \multirow[t]{2}{*}{ Clinoptilo } & \multirow{2}{*}{ K-Feldspar } & \multirow{2}{*}{\begin{tabular}{|c|} 
Plagioclase \\
$10 \%$
\end{tabular}} & \multirow[t]{2}{*}{ Pyrite } \\
\hline Number & Depth & & 10 & & & & & & & & \\
\hline 2 & & 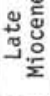 & & & & & & & & & \\
\hline 3 & & 㻤 & & & & & & & & & \\
\hline 4 & & $\stackrel{\circ}{\tilde{g}}$ & & & & & & & & & \\
\hline 5 & & 悫 & & & & & & & & & \\
\hline & $496-$ & & & & & & & & & & \\
\hline
\end{tabular}

Figure 4. Hole 62, X-ray results, composited less than $2 \mu$ samples. 


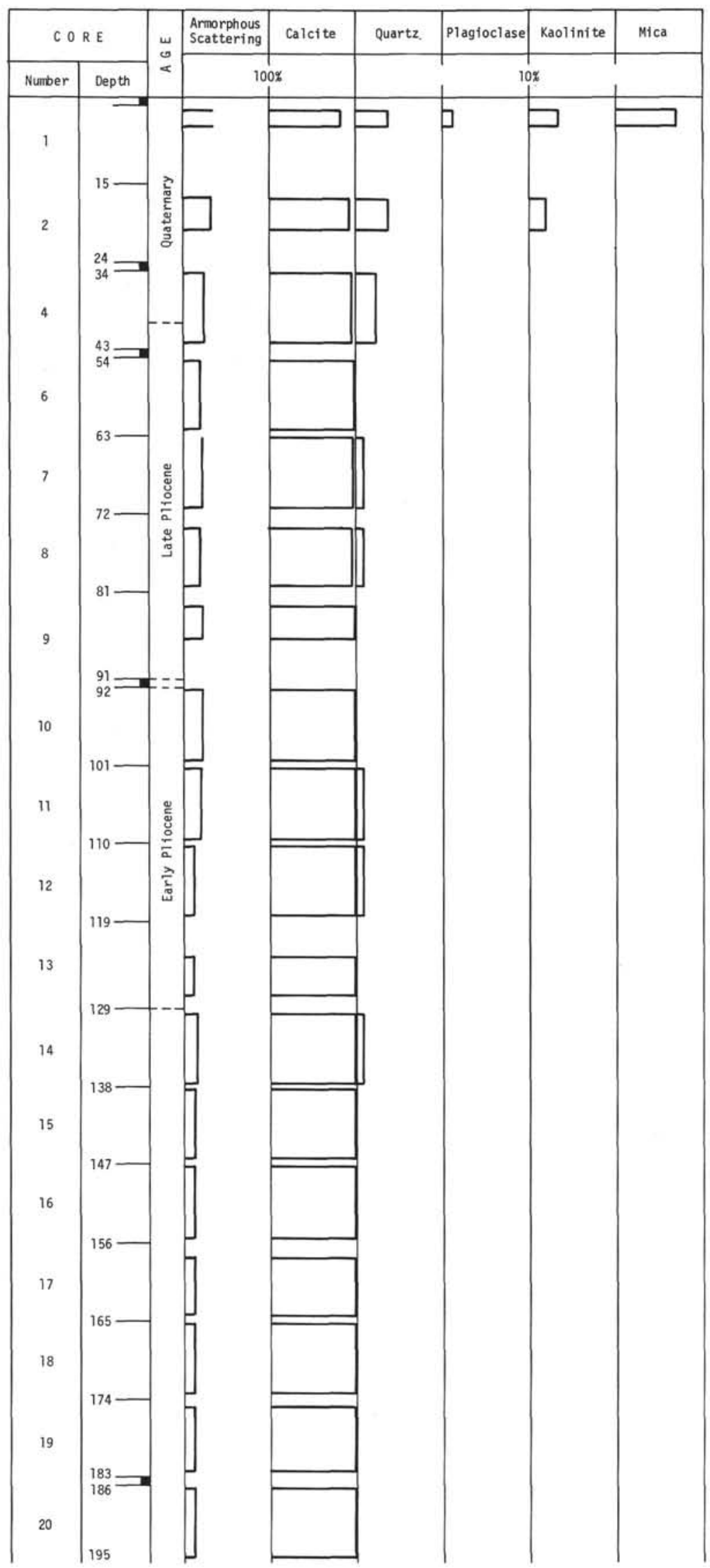

Figure 5. Hole 62A, X-ray results, composited bulk samples. 


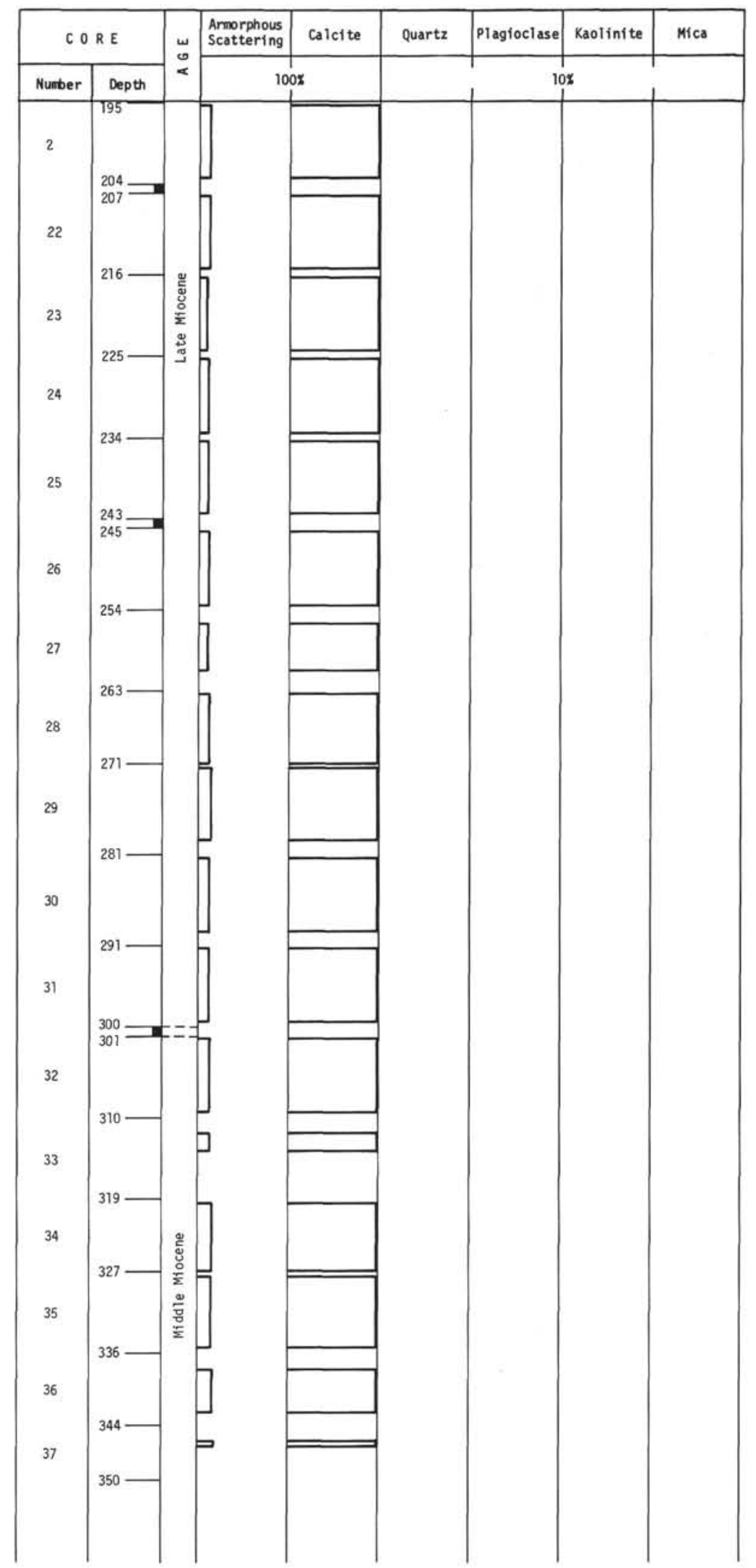

Figure 5. Continued. 


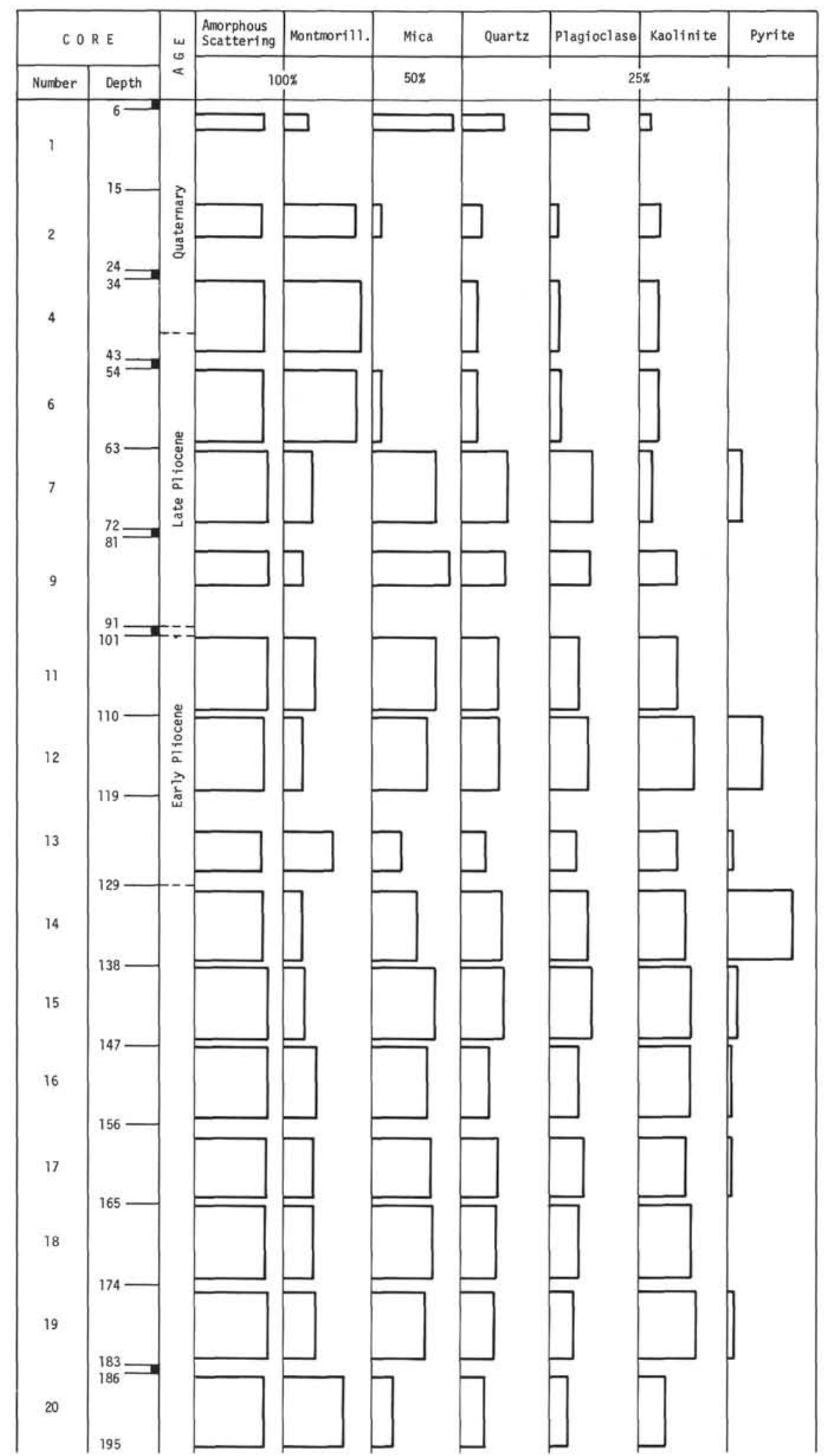

Figure 6. Hole $62 A, X$-ray results, composited less than $2 \mu$ samples. 


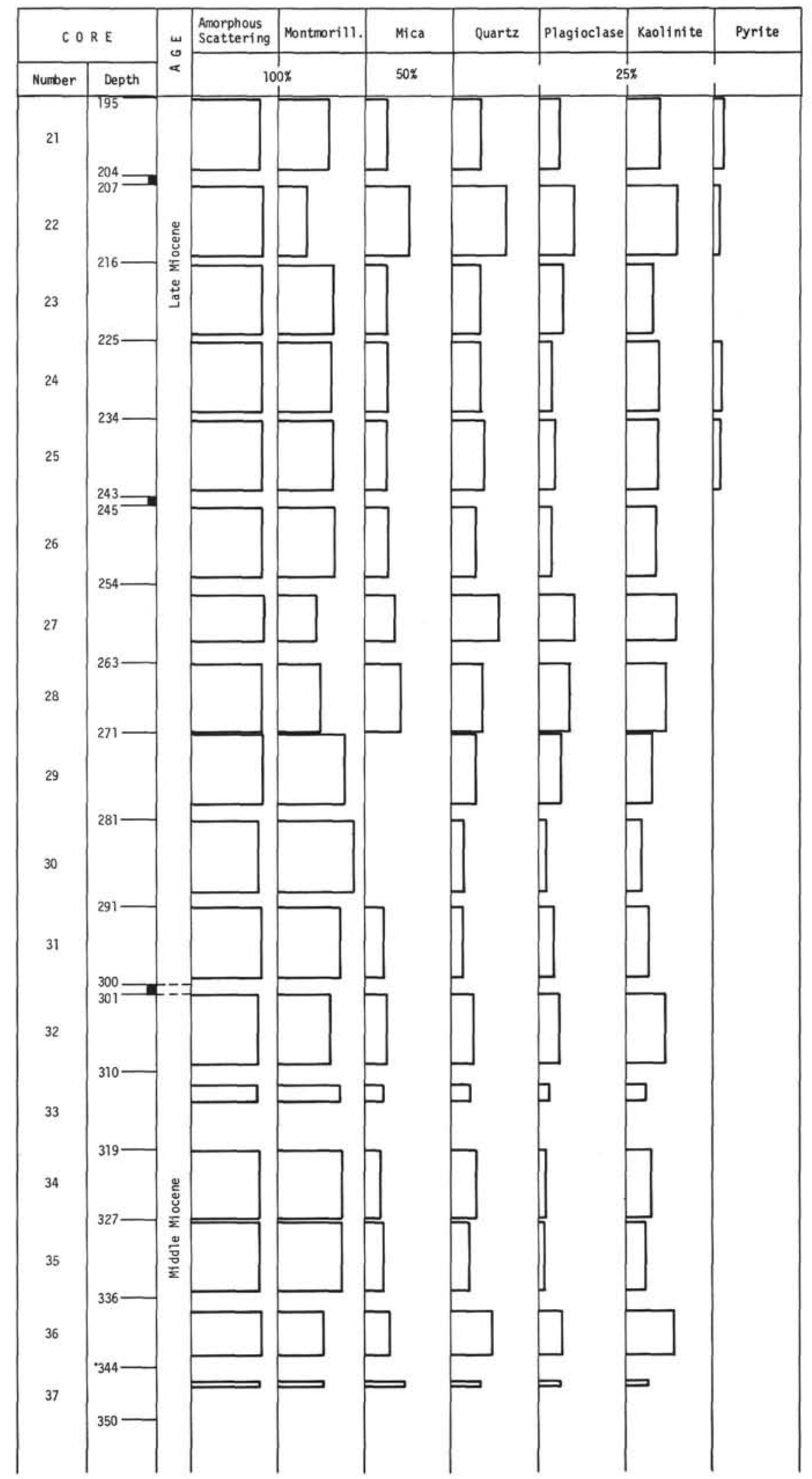

Figure 6. Continued. 


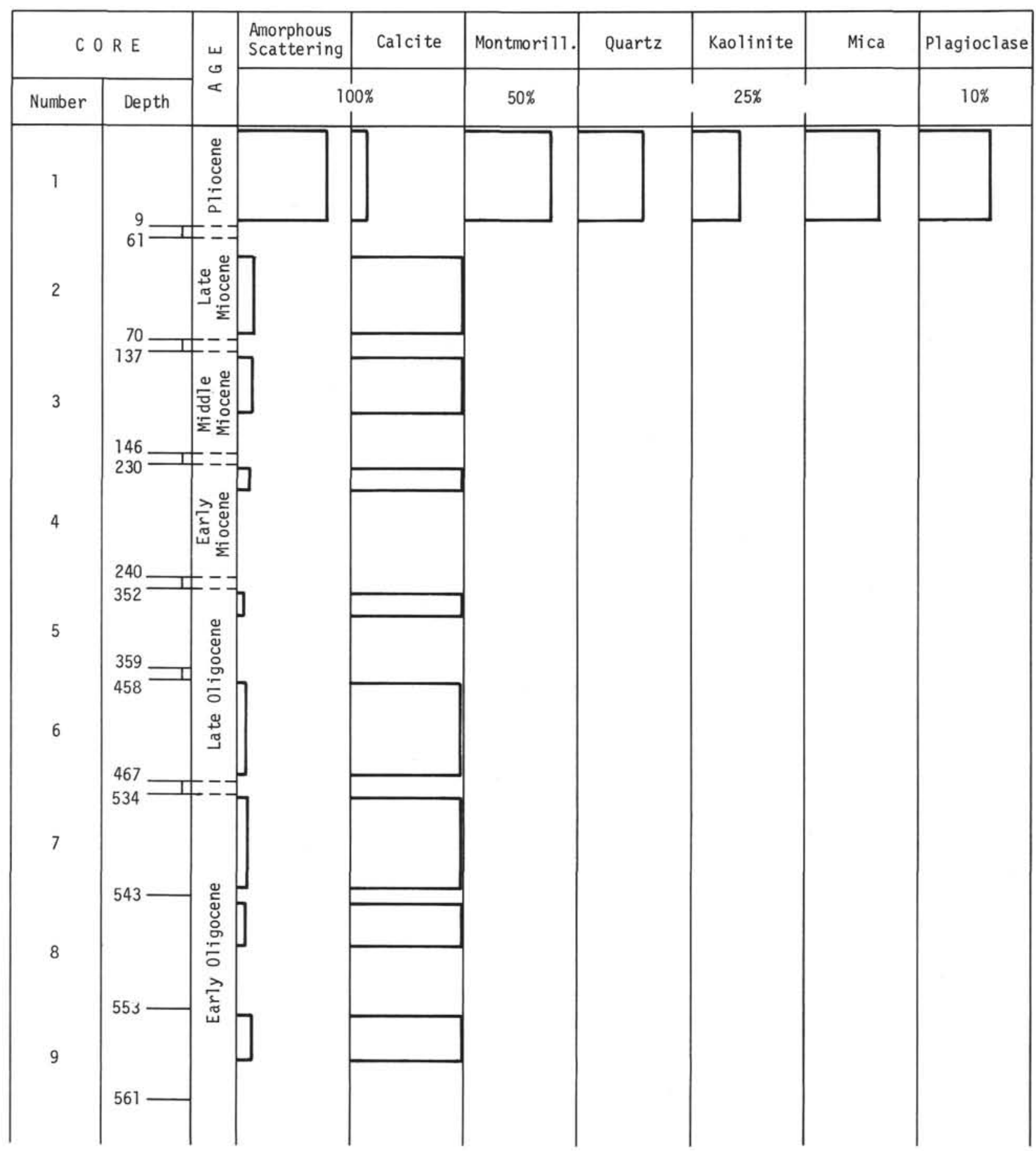

Figure 7. Hole 63, X-ray results, composited bulk samples. 


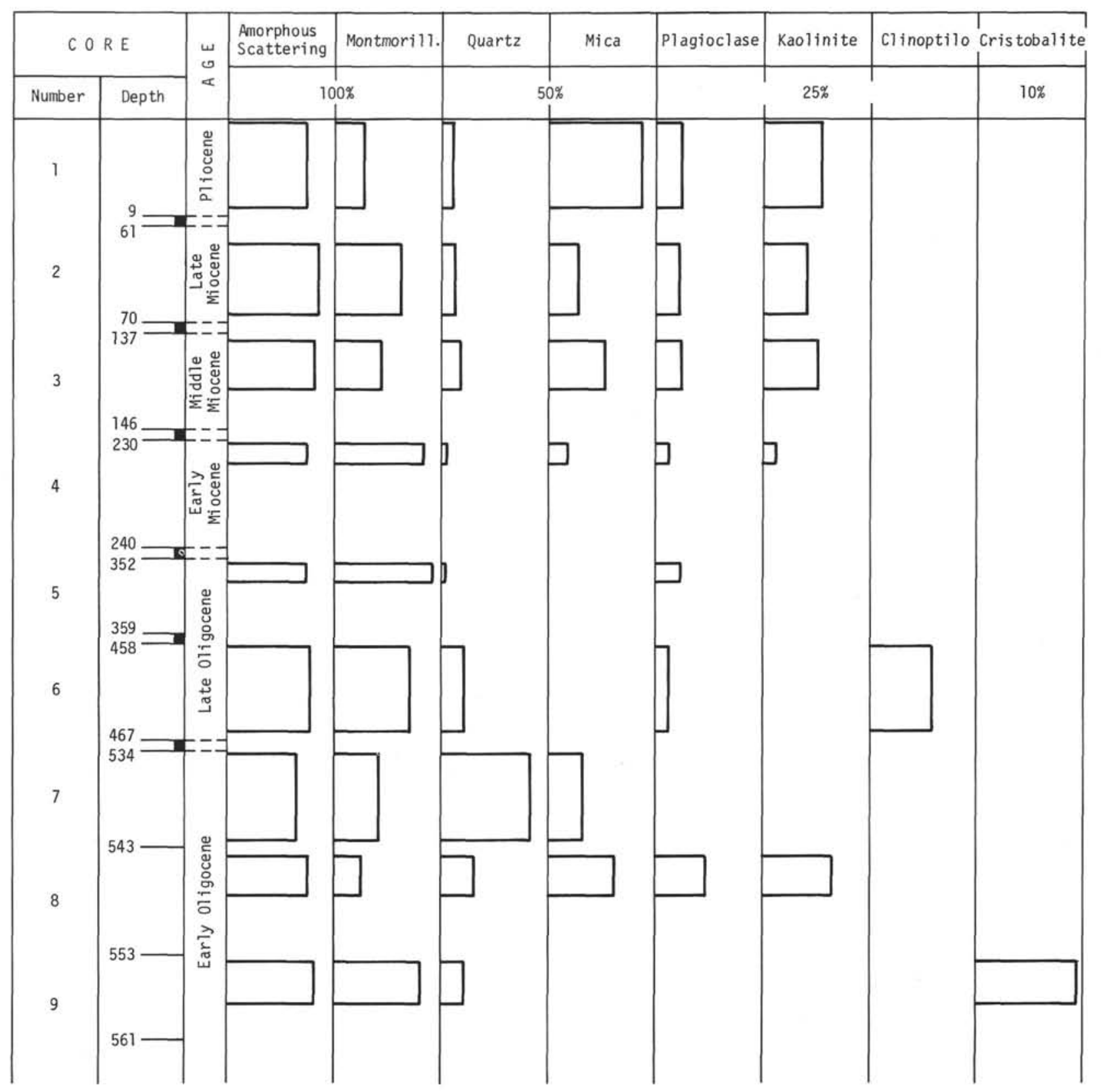

Figure 8. Hole 63, $X$-ray results, composited less than $2 \mu$ samples. 


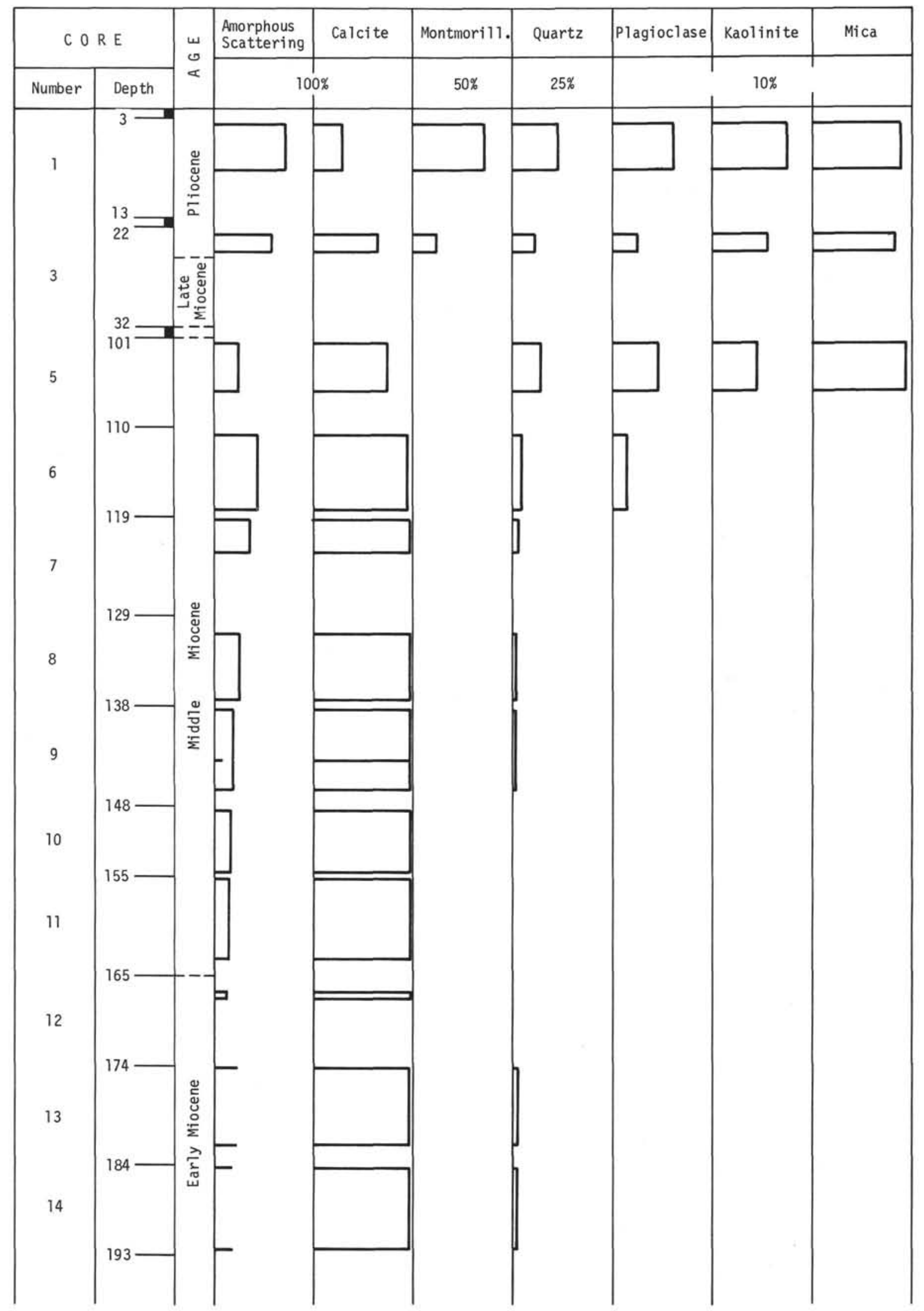

Figure 9. Hole 63A, X-ray results, composited bulk samples. 


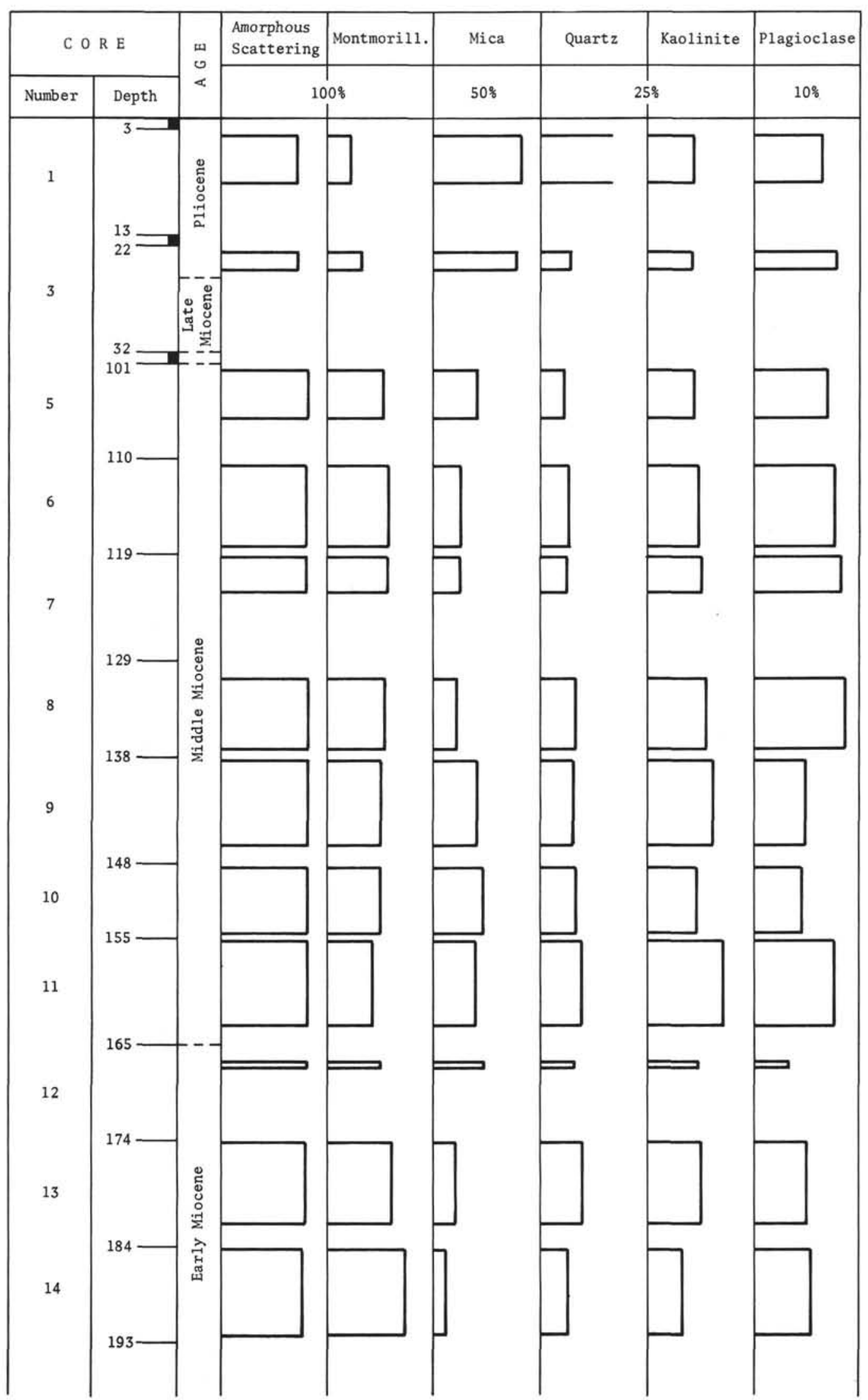

Figure 10. Hole $63 A, X$-ray results, composited less than $62 \mu$ samples. 


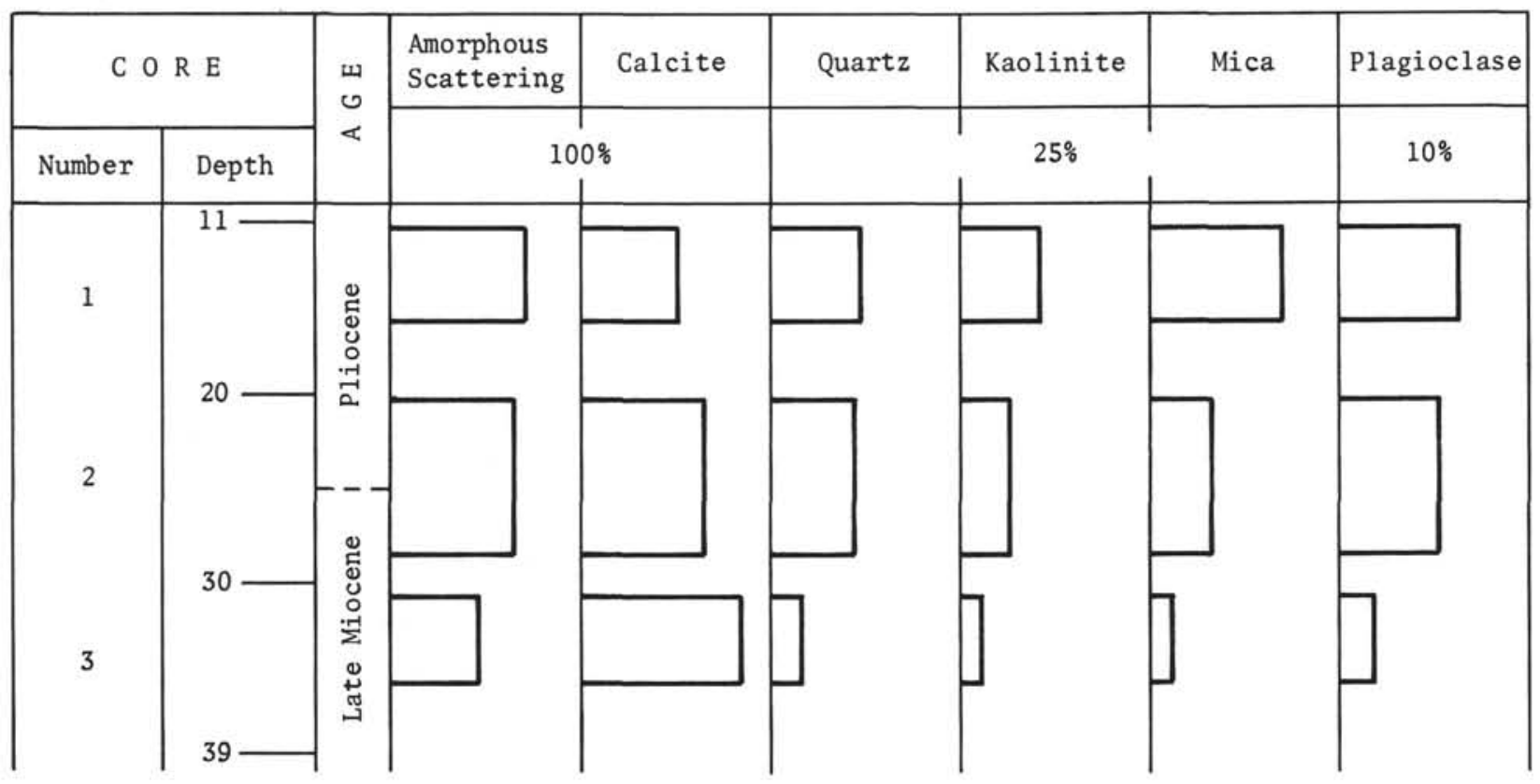

Figure 11. Hole 63B, X-ray results, composited bulk samples.

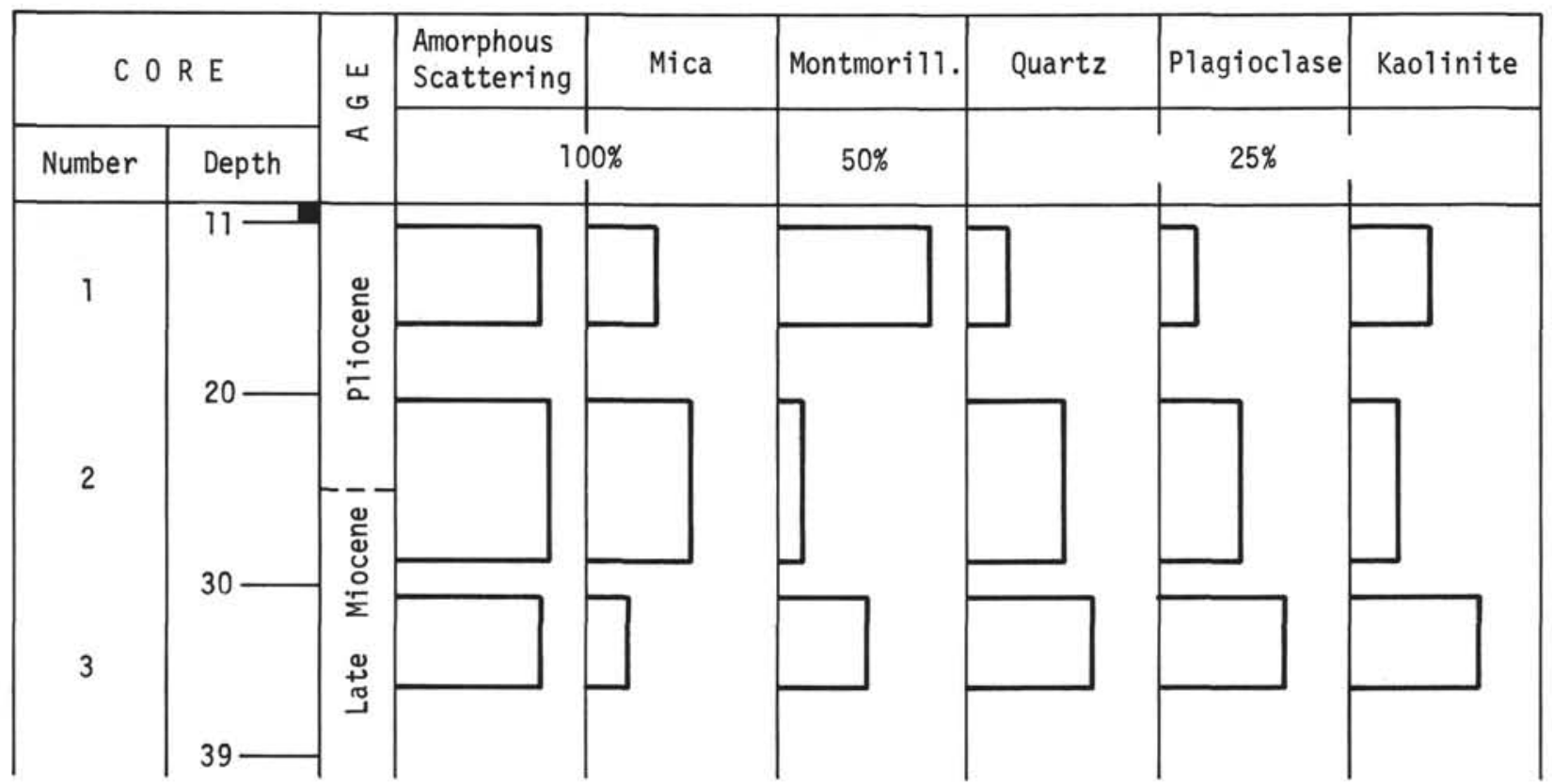

Figure 12. Hole $64, X$-ray results, composited less than $2 \mu$ samples. 


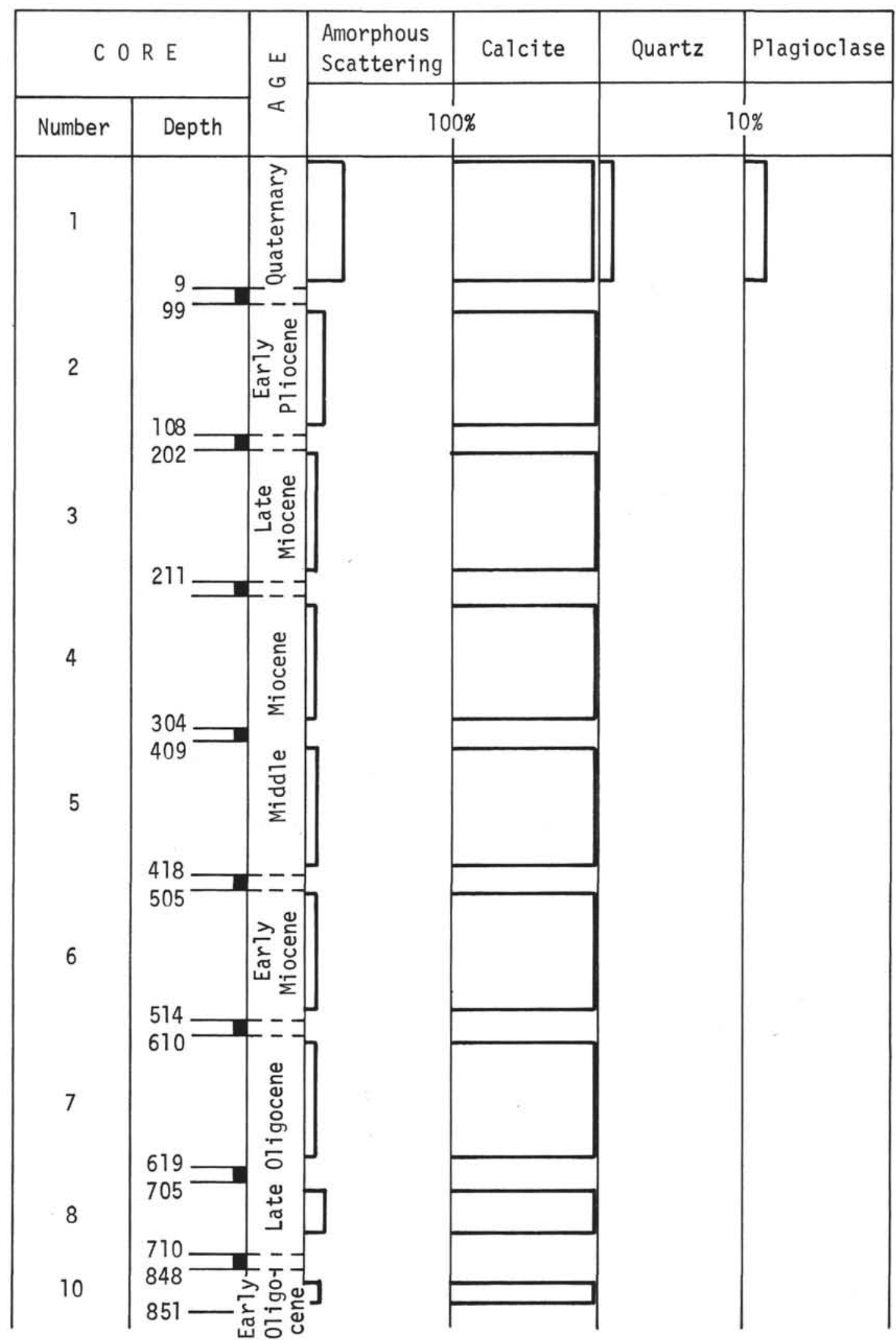

Figure 13. Hole 64, X-ray results, composited bulk samples. 


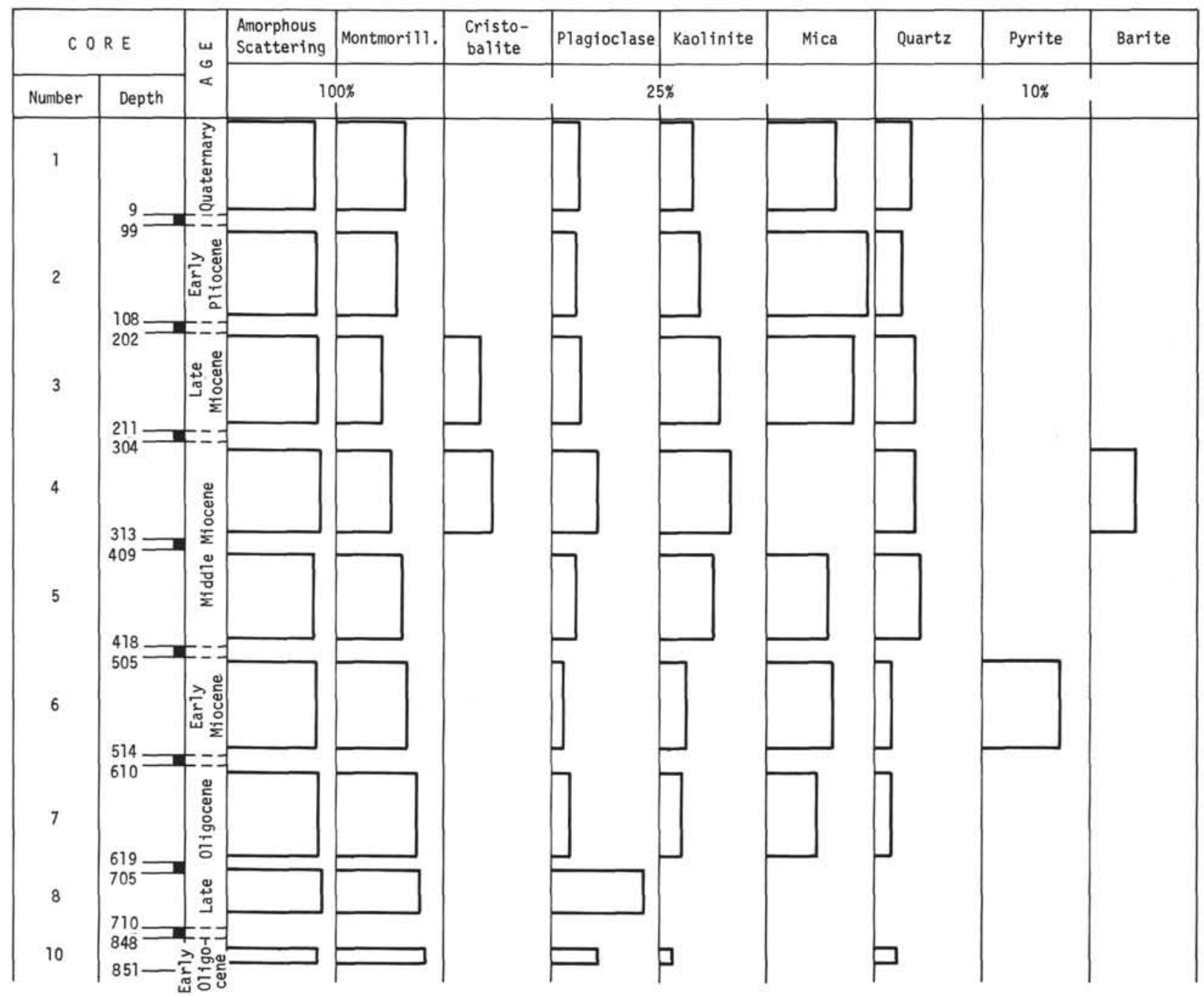

Figure 14 . Hole $64, X$-ray results, composited less than $2 \mu$ samples. 


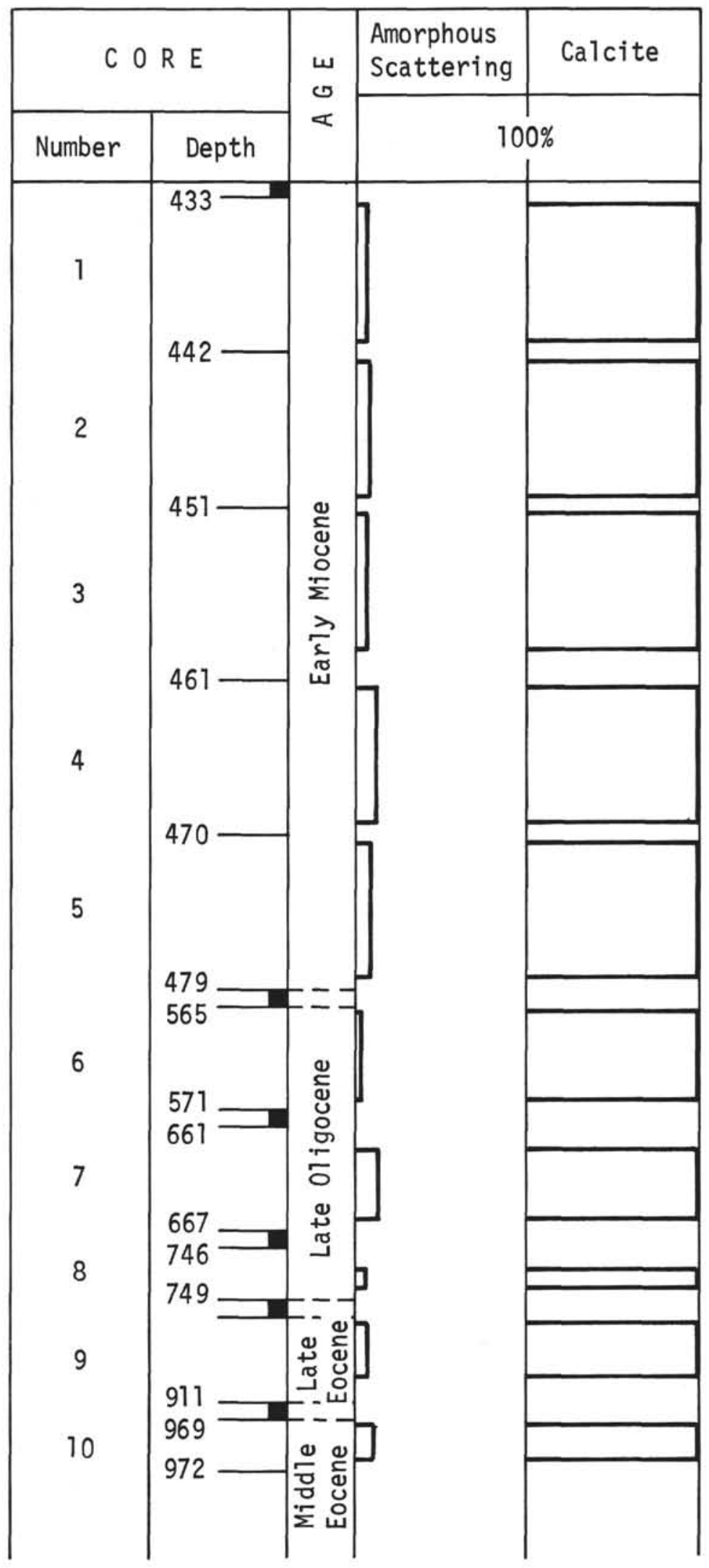

Figure 15. Hole 64A, $X$-ray results, composited bulk samples. 


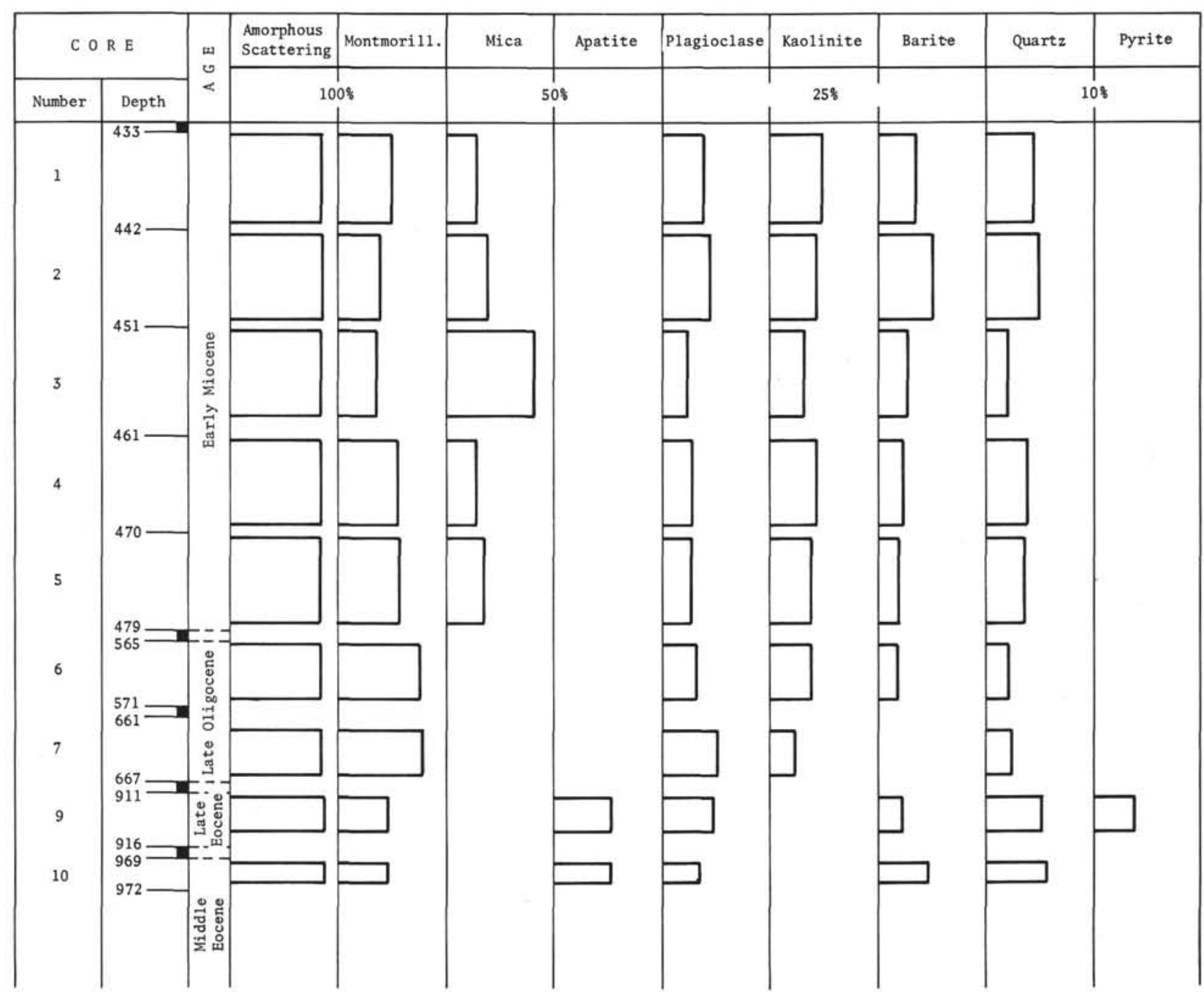

Figure 16. Hole $64 A, X$-ray results, composited less than $2 \mu$ samples. 


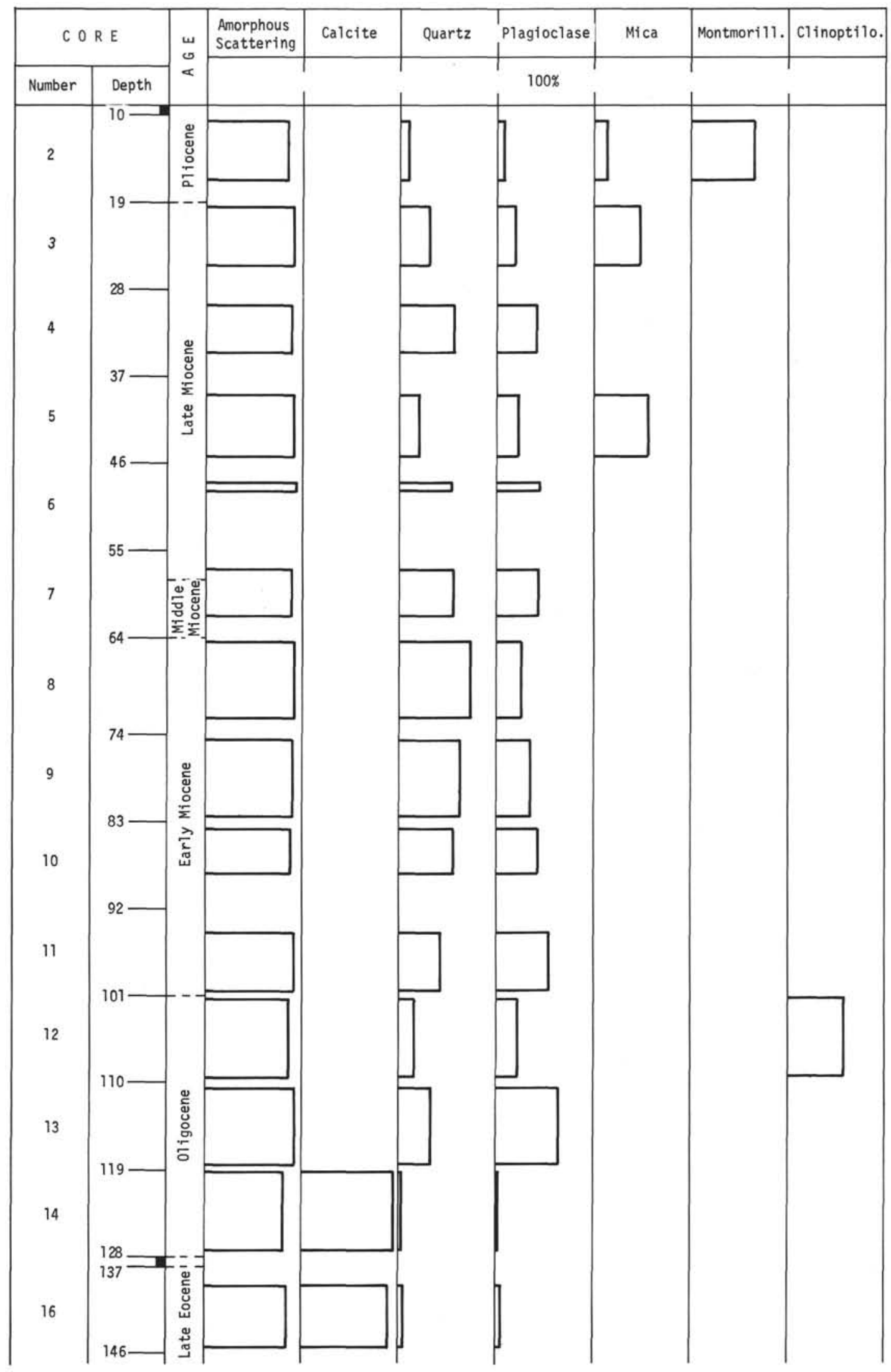

Figure 17. Hole 65, X-ray results, composited bulk samples. 


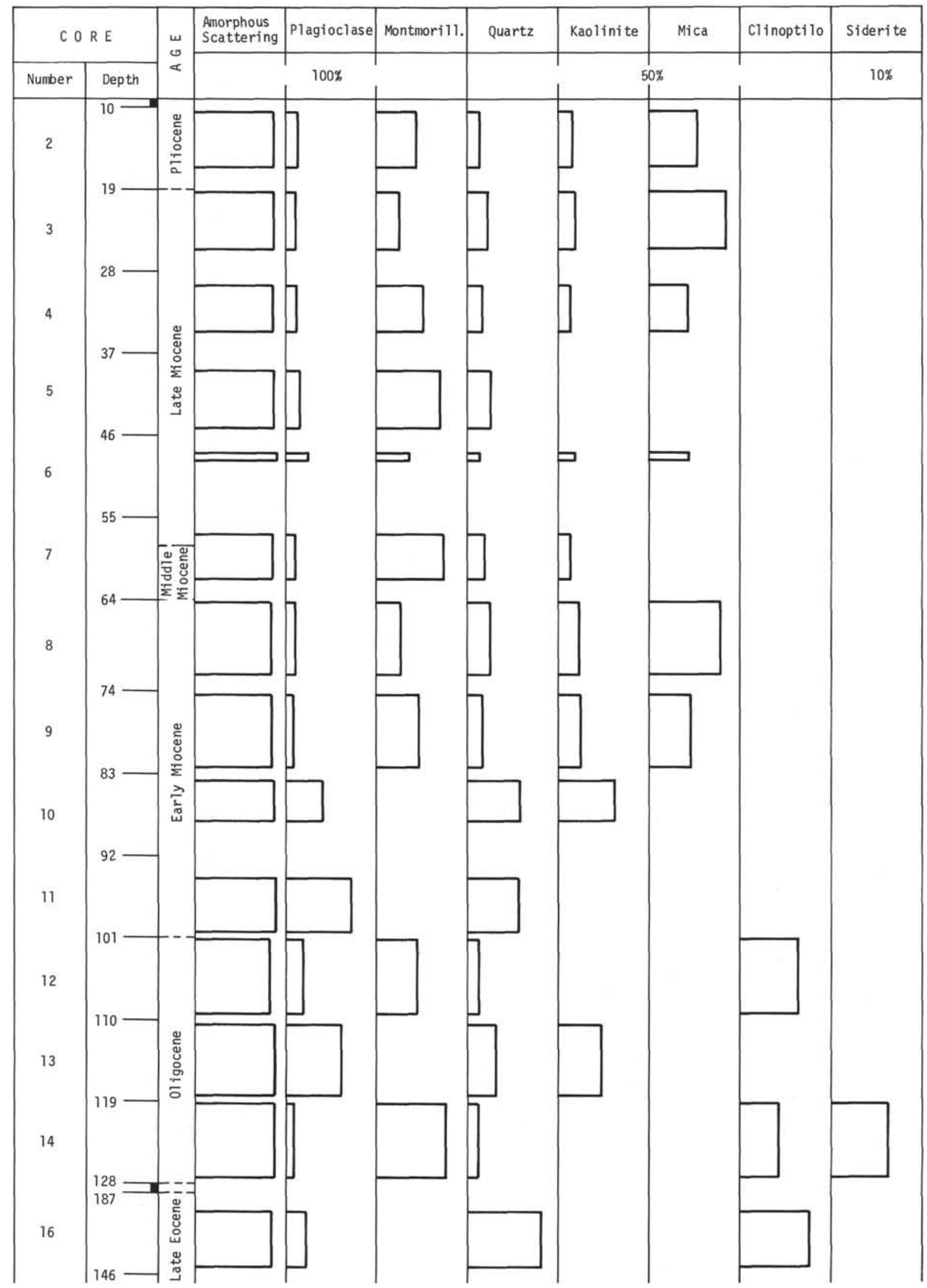

Figure 18. Hole $65, X$-ray results, composited less than $2 \mu$ samples. 


\begin{tabular}{|c|c|c|c|c|c|c|c|c|}
\hline \multicolumn{2}{|c|}{$C O R E$} & \multirow{2}{*}{$\begin{array}{l}w \\
0 \\
\alpha\end{array}$} & $\begin{array}{l}\text { Amorphous } \\
\text { Scattering }\end{array}$ & Calcite & Clinoptilo. & Montmorill. & Quartz & Plagioclase \\
\hline Number & Depth & & \multicolumn{3}{|c|}{$100 \%$} & & \multicolumn{2}{|c|}{$10 \%$} \\
\hline 2 & & ơ & & & & & & $=$ \\
\hline 4 & & 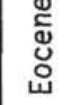 & & & & & & \\
\hline 5 & 168 & $\frac{\pi}{2}$ & & & & & & \\
\hline
\end{tabular}

Figure 19. Hole 65A, X-ray results, composited bulk samples.

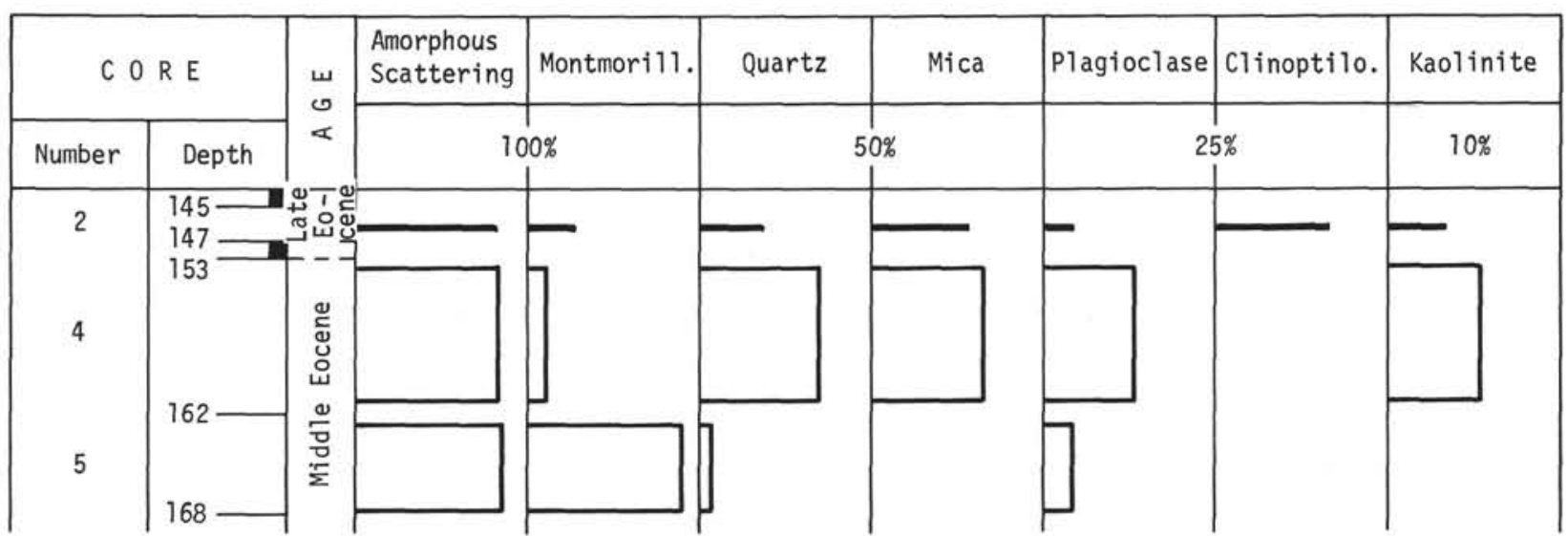

Figure 20. Hole 65A, X-ray results, composited less than $2 \mu$ samples. 


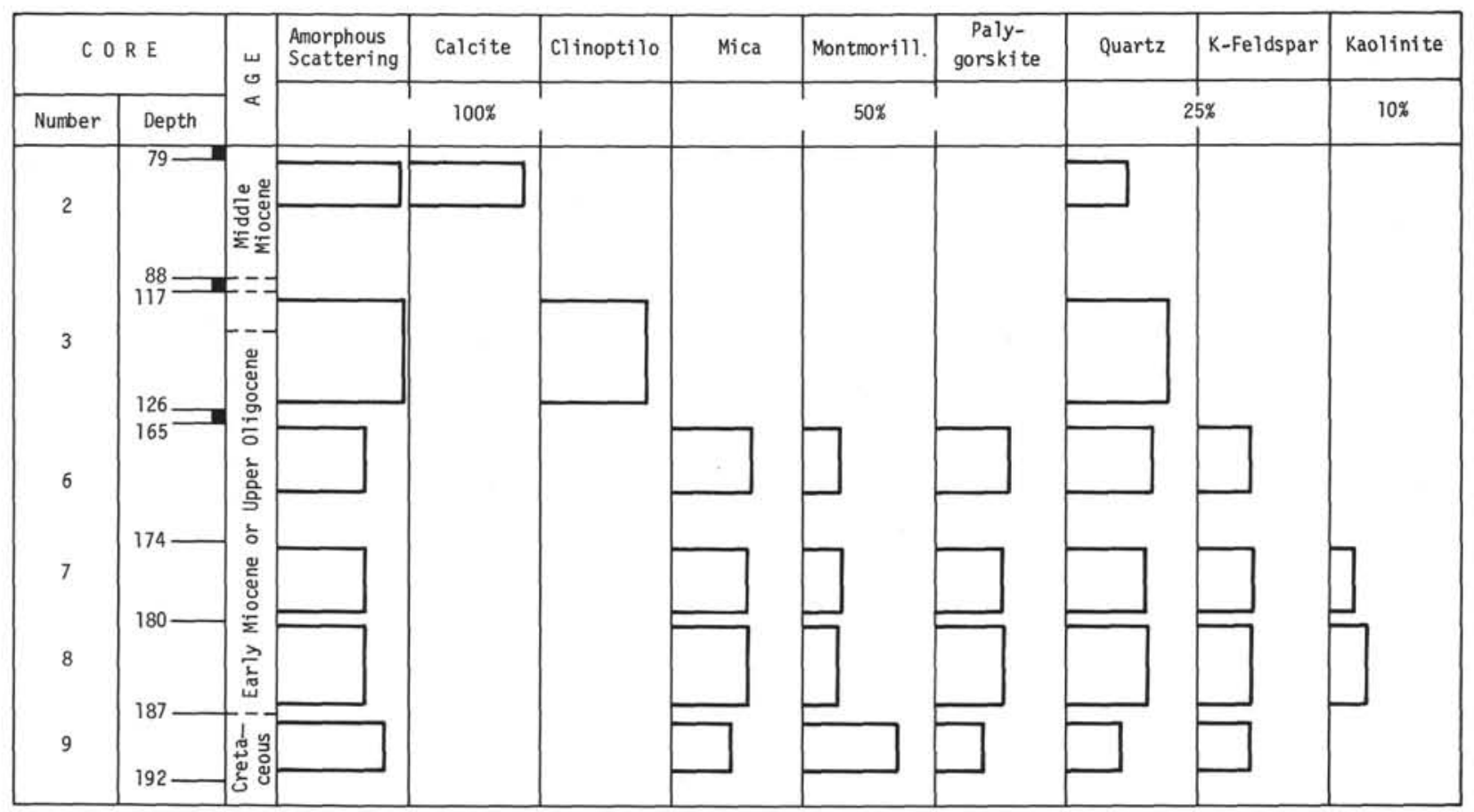

Figure 21. Hole 66, X-ray results, composited bulk samples.

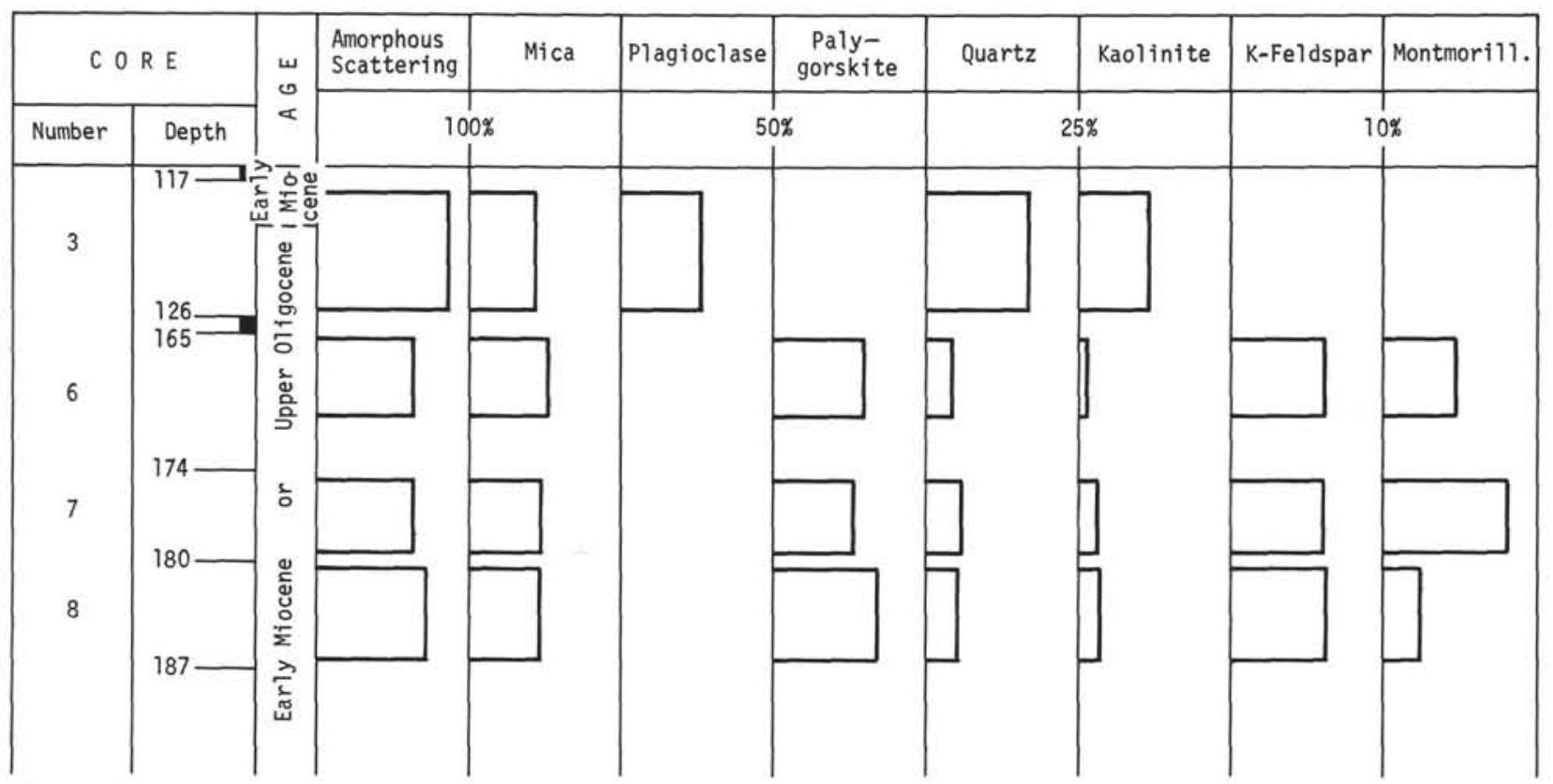

Figure 22. Hole 66, X-ray results, composited less than $2 \mu$ samples. 


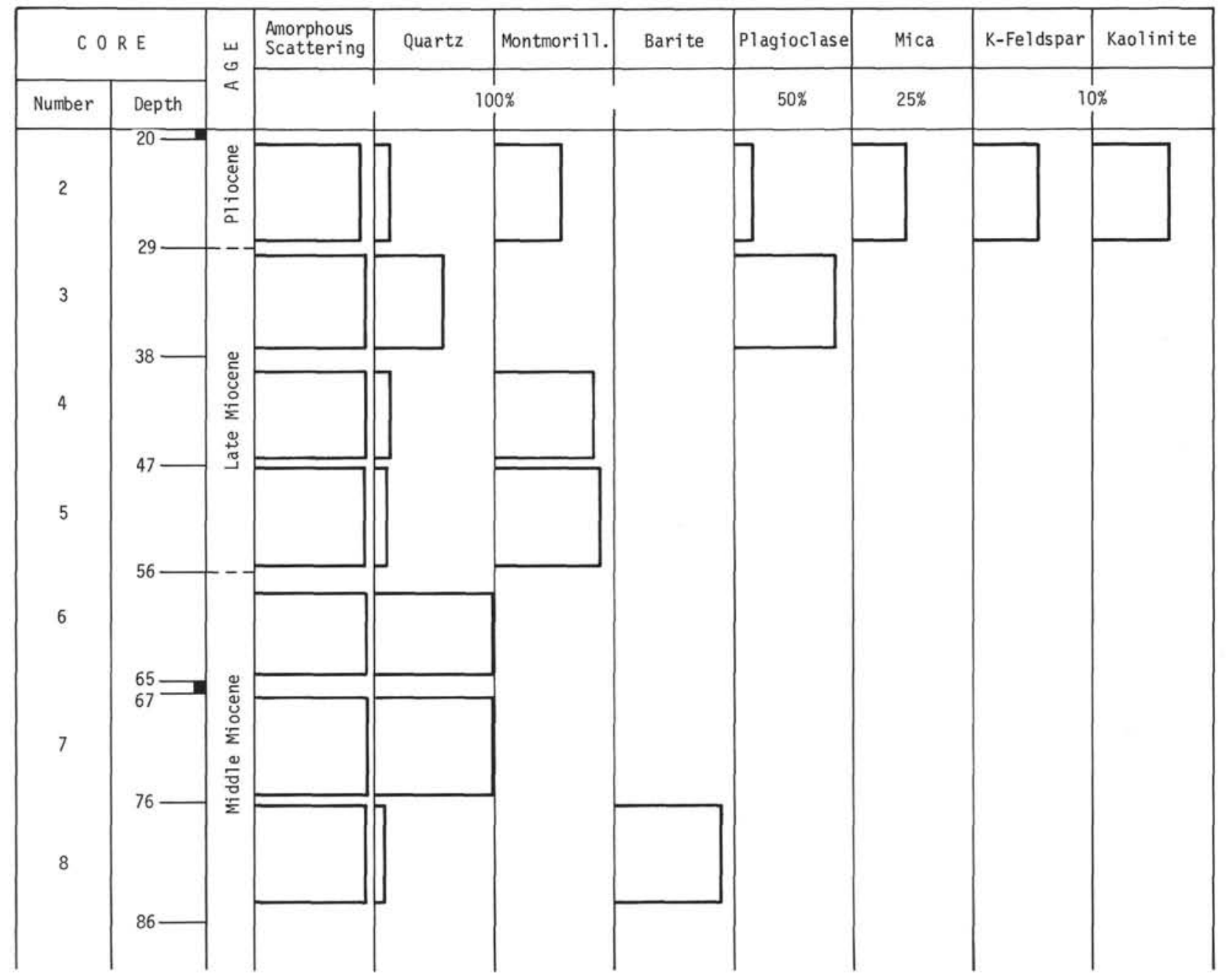

Figure 23. Hole 66A, X-ray results, composited bulk samples. 


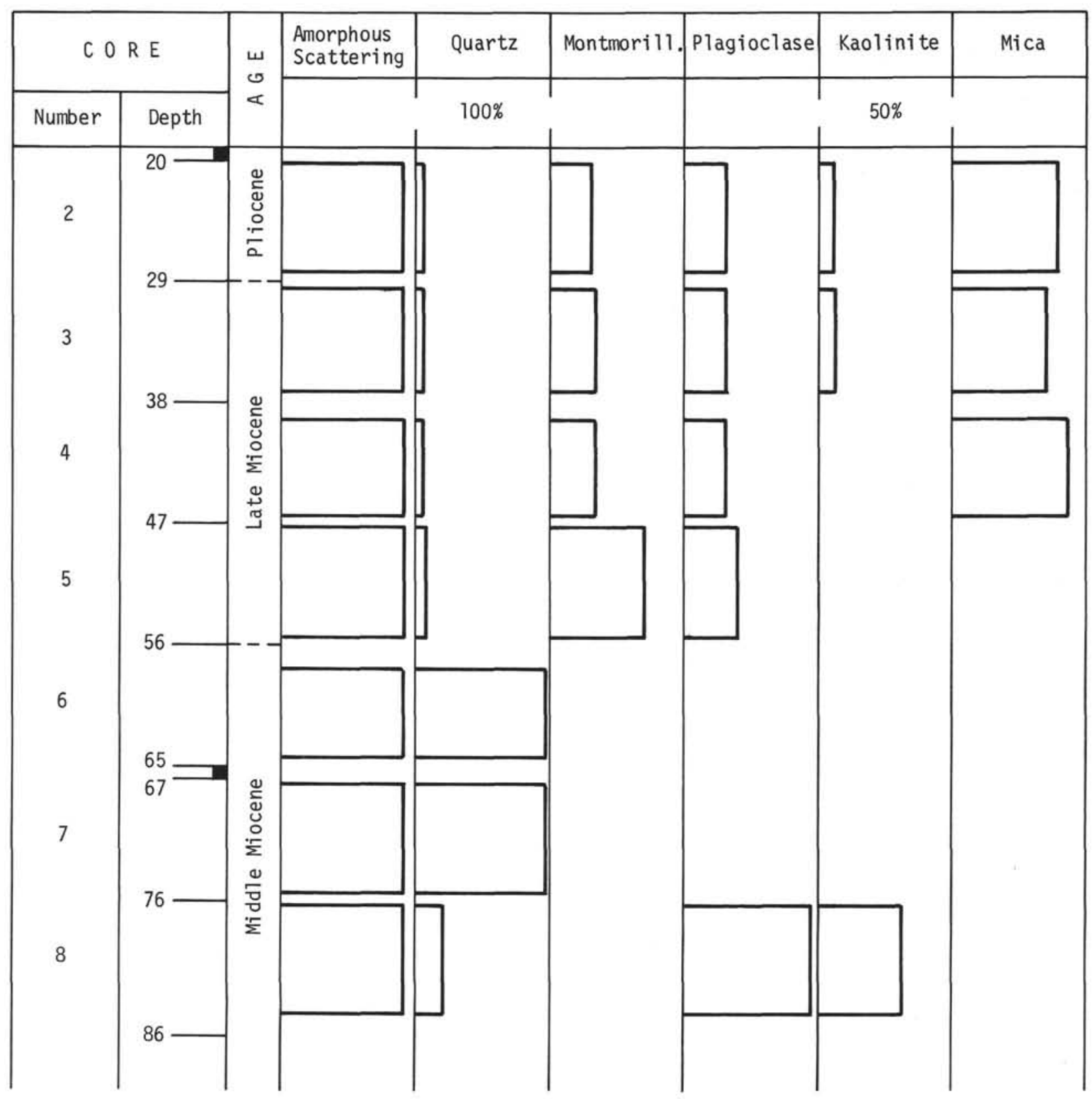

Figure 24 . Hole $66 \mathrm{~A}, \mathrm{X}$-ray results, composited less than $2 \mu$ samples. 


\begin{tabular}{|c|c|c|c|c|c|c|}
\hline \multicolumn{2}{|c|}{$C O R E$} & \multirow{2}{*}{$\begin{array}{l}\omega \\
\sigma \\
\sigma\end{array}$} & $\begin{array}{l}\text { Amorphous } \\
\text { Scattering }\end{array}$ & Phillipsite & Plagioclase & Quartz \\
\hline Number & Depth & & & $0 \%$ & $25 \%$ & $10 \%$ \\
\hline 1 & $5-$ & 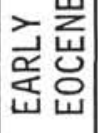 & & & & \\
\hline
\end{tabular}

Figure 25. Hole 67, X-ray results, composited bulk samples.

\begin{tabular}{|c|c|c|c|c|c|c|c|c|}
\hline \multicolumn{2}{|c|}{$C O R E$} & س & $\begin{array}{l}\text { Amorphous } \\
\text { Scattering }\end{array}$ & Mica & Phillipsite & Plagioclase & Quartz & Kaolinite \\
\hline Number & Depth & $\alpha$ & $100 \%$ & & $0 \%$ & $25 \%$ & 10 & \\
\hline 1 & 5 & 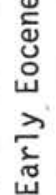 & & & & & & \\
\hline
\end{tabular}

Figure 26. Hole $67, X$-ray results, composited less than $2 \mu$ samples. 


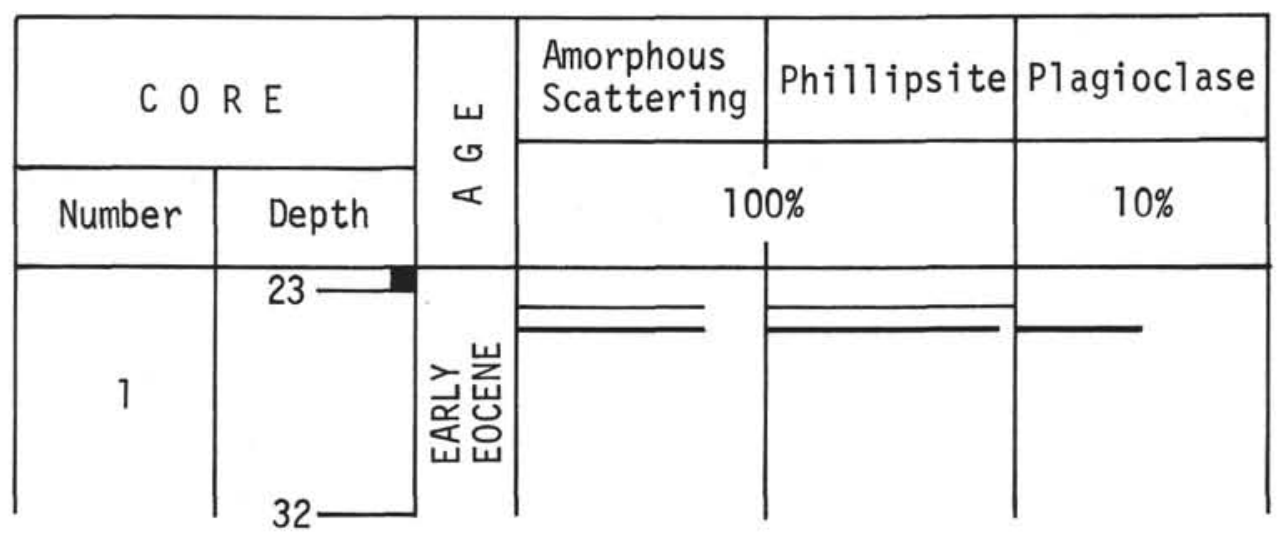

Figure 27. Hole 67A, X-ray results, composited bulk samples.

\begin{tabular}{|c|c|c|c|c|c|c|}
\hline \multicolumn{2}{|c|}{$C O R E$} & \multirow{2}{*}{$\begin{array}{l}\omega \\
\sigma \\
\ll\end{array}$} & $\begin{array}{l}\text { Amorphous } \\
\text { Scattering }\end{array}$ & Montmorill. & Plagioclase & Kaolinite \\
\hline Number & Depth & & & $0 \%$ & $25 \%$ & $10 \%$ \\
\hline 1 & $\begin{array}{l}23- \\
32\end{array}$ & 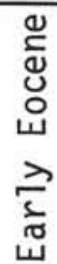 & & & & \\
\hline
\end{tabular}

Figure 28. Hole 67A, X-ray results, composited less than $2 \mu$ samples. 
TABLE 1

Results of X-Ray Diffraction Analysis of Composited Bulk Samples from Leg $7^{\mathrm{a}}$

\begin{tabular}{|c|c|c|c|c|c|c|c|c|c|c|c|c|c|c|c|c|}
\hline \multicolumn{17}{|c|}{ Hole 61.1} \\
\hline Core & $\begin{array}{l}\text { Downhole } \\
\text { Depth of } \\
\text { Core }\end{array}$ & $\begin{array}{c}\text { Depth } \\
\text { Range } \\
\text { Sampled }\end{array}$ & Diff. & $\begin{array}{c}\% \\
\text { Amorphous }\end{array}$ & Calc. & Quar. & Plag. & Kaol. & Mica & Mont. & Clin. & $\mathrm{K}-\mathrm{Fe}$ & Paly. & Barite & Phil. & Crist. \\
\hline 1 & $83-89$ & $\begin{array}{l}84.04- \\
85.82\end{array}$ & 77.4 & 33.5 & 0.0 & 9.5 & 1.3 & 0.0 & 0.0 & 0.0 & 0.0 & 0.0 & 0.0 & 0.0 & 0.0 & 89.2 \\
\hline \multicolumn{17}{|c|}{ Hole 62.0} \\
\hline Core & $\begin{array}{l}\text { Downhole } \\
\text { Depth of } \\
\text { Core }\end{array}$ & $\begin{array}{c}\text { Depth } \\
\text { Range } \\
\text { Sampled }\end{array}$ & Diff. & $\begin{array}{c}\% \\
\text { Amorphous }\end{array}$ & Calc. & Quar. & Plag. & Kaol. & Mica & Mont. & Clin. & $\mathrm{K}-\mathrm{Fe}$ & Paly. & Barite & Phil. & Crist. \\
\hline 1 & $91-100$ & $\begin{array}{l}91.04- \\
99.21\end{array}$ & 72.6 & 19.4 & 100.0 & 0.0 & 0.0 & 0.0 & 0.0 & 0.0 & 0.0 & 0.0 & 0.0 & 0.0 & 0.0 & 0.0 \\
\hline 2 & 205-214 & $\begin{array}{l}205.89- \\
213.69\end{array}$ & 70.6 & 13.5 & 100.0 & 0.0 & 0.0 & 0.0 & 0.0 & 0.0 & 0.0 & 0.0 & 0.0 & 0.0 & 0.0 & 0.0 \\
\hline 3 & 299-308 & $\begin{array}{l}299.04- \\
307.21\end{array}$ & 71.6 & 16.5 & 100.0 & 0.0 & 0.0 & 0.0 & 0.0 & 0.0 & 0.0 & 0.0 & 0.0 & 0.0 & 0.0 & 0.0 \\
\hline 4 & $395-401$ & $\begin{array}{l}395.08- \\
403.23\end{array}$ & 72.9 & 20.3 & 100.0 & 0.0 & 0.0 & 0.0 & 0.0 & 0.0 & 0.0 & 0.0 & 0.0 & 0.0 & 0.0 & 0.0 \\
\hline 5 & $490-496$ & $\begin{array}{l}491.67- \\
495.23\end{array}$ & 71.1 & 15.0 & 100.0 & 0.0 & 0.0 & 0.0 & 0.0 & 0.0 & 0.0 & 0.0 & 0.0 & 0.0 & 0.0 & 0.0 \\
\hline \multicolumn{17}{|c|}{ Hole 62.1} \\
\hline Core & $\begin{array}{l}\text { Downhole } \\
\text { Depth of } \\
\text { Core }\end{array}$ & $\begin{array}{c}\text { Depth } \\
\text { Range } \\
\text { Sampled }\end{array}$ & Diff. & $\begin{array}{c}\% \\
\text { Amorphous }\end{array}$ & Calc. & Quar. & Plag. & Kaol. & Mica & Mont. & Clin. & $\mathrm{K}-\mathrm{Fe}$ & Paly. & Barite & Phil. & Crist. \\
\hline 1 & $6-15$ & $\begin{array}{l}6.33- \\
8.23\end{array}$ & 78.1 & 35.6 & 84.2 & 3.9 & 1.3 & 3.5 & 7.0 & 0.0 & 0.0 & 0.0 & 0.0 & 0.0 & 0.0 & 0.0 \\
\hline 2 & $15-24$ & $\begin{array}{l}16.55- \\
20.25\end{array}$ & 77.1 & 32.6 & 93.8 & 3.9 & 0.0 & 2.0 & 0.0 & 0.0 & 0.0 & 0.0 & 0.0 & 0.0 & 0.0 & 0.0 \\
\hline 4 & $34-43$ & $\begin{array}{l}34.05- \\
42.21\end{array}$ & 74.9 & 26.2 & 97.5 & 2.5 & 0.0 & 0.0 & 0.0 & 0.0 & 0.0 & 0.0 & 0.0 & 0.0 & 0.0 & 0.0 \\
\hline
\end{tabular}


TABLE 1 - Continued

\begin{tabular}{|c|c|c|c|c|c|c|c|c|c|c|c|c|c|c|c|c|}
\hline \multicolumn{17}{|c|}{ Hole 62.1 - Continued } \\
\hline Core & $\begin{array}{l}\text { Downhole } \\
\text { Depth of } \\
\text { Core }\end{array}$ & $\begin{array}{c}\text { Depth } \\
\text { Range } \\
\text { Sampled }\end{array}$ & Diff. & $\begin{array}{c}\% \\
\text { Amorphous }\end{array}$ & Calc. & Quar. & Plag. & Kaol. & Mica & Mont. & Clin. & $\mathrm{K}-\mathrm{Fe}$ & Paly. & Barite & Phil. & Crist. \\
\hline 6 & $54-63$ & $\begin{array}{l}54.13- \\
62.21\end{array}$ & 73.3 & 21.5 & 100.0 & 0.0 & 0.0 & 0.0 & 0.0 & 0.0 & 0.0 & 0.0 & 0.0 & 0.0 & 0.0 & 0.0 \\
\hline 7 & $63-72$ & $\begin{array}{l}63.05- \\
71.21\end{array}$ & 74.1 & 23.8 & 99.0 & 1.0 & 0.0 & 0.0 & 0.0 & 0.0 & 0.0 & 0.0 & 0.0 & 0.0 & 0.0 & 0.0 \\
\hline 8 & $72-81$ & $\begin{array}{l}73.56- \\
80.21\end{array}$ & 73.2 & 21.2 & 99.0 & 1.0 & 0.0 & 0.0 & 0.0 & 0.0 & 0.0 & 0.0 & 0.0 & 0.0 & 0.0 & 0.0 \\
\hline 9 & $81-91$ & $\begin{array}{l}82.55- \\
86.21\end{array}$ & 74.1 & 23.8 & 100.0 & 0.0 & 0.0 & 0.0 & 0.0 & 0.0 & 0.0 & 0.0 & 0.0 & 0.0 & 0.0 & 0.0 \\
\hline 10 & $92-101$ & $\begin{array}{c}92.05- \\
100.21\end{array}$ & 74.2 & 24.1 & 100.0 & 0.0 & 0.0 & 0.0 & 0.0 & 0.0 & 0.0 & 0.0 & 0.0 & 0.0 & 0.0 & 0.0 \\
\hline 11 & $101-110$ & $\begin{array}{l}101.05- \\
109.48\end{array}$ & 73.6 & 22.4 & 99.0 & 1.0 & 0.0 & 0.0 & 0.0 & 0.0 & 0.0 & 0.0 & 0.0 & 0.0 & 0.0 & 0.0 \\
\hline 12 & $110-119$ & $\begin{array}{l}110.06- \\
118.21\end{array}$ & 71.0 & 14.7 & 99.0 & 1.0 & 0.0 & 0.0 & 0.0 & 0.0 & 0.0 & 0.0 & 0.0 & 0.0 & 0.0 & 0.0 \\
\hline 13 & $119-129$ & $\begin{array}{l}122.94- \\
127.25\end{array}$ & 70.8 & 14.1 & 100.0 & 0.0 & 0.0 & 0.0 & 0.0 & 0.0 & 0.0 & 0.0 & 0.0 & 0.0 & 0.0 & 0.0 \\
\hline 14 & $129-138$ & $\begin{array}{l}129.55- \\
137.66\end{array}$ & 72.2 & 18.2 & 99.0 & 1.0 & 0.0 & 0.0 & 0.0 & 0.0 & 0.0 & 0.0 & 0.0 & 0.0 & 0.0 & 0.0 \\
\hline 15 & $138-147$ & $\begin{array}{l}138.05- \\
146.21\end{array}$ & 71.3 & 15.6 & 100.0 & 0.0 & 0.0 & 0.0 & 0.0 & 0.0 & 0.0 & 0.0 & 0.0 & 0.0 & 0.0 & 0.0 \\
\hline 16 & $147-156$ & $\begin{array}{l}147.06- \\
155.21\end{array}$ & 70.9 & 14.4 & 100.0 & 0.0 & 0.0 & 0.0 & 0.0 & 0.0 & 0.0 & 0.0 & 0.0 & 0.0 & 0.0 & 0.0 \\
\hline 17 & $156-165$ & $\begin{array}{l}157.26- \\
164.18\end{array}$ & 71.2 & 15.3 & 100.0 & 0.0 & 0.0 & 0.0 & 0.0 & 0.0 & 0.0 & 0.0 & 0.0 & 0.0 & 0.0 & 0.0 \\
\hline 18 & $165-174$ & $\begin{array}{l}165.05- \\
173.19\end{array}$ & 71.2 & 15.3 & 100.0 & 0.0 & 0.0 & 0.0 & 0.0 & 0.0 & 0.0 & 0.0 & 0.0 & 0.0 & 0.0 & 0.0 \\
\hline 19 & $174-183$ & $\begin{array}{l}174.82- \\
182.21\end{array}$ & 71.1 & 15.0 & 100.0 & 0.0 & 0.0 & 0.0 & 0.0 & 0.0 & 0.0 & 0.0 & 0.0 & 0.0 & 0.0 & 0.0 \\
\hline 20 & $186-195$ & $\begin{array}{l}186.13- \\
194.21\end{array}$ & 71.1 & 15.0 & 100.0 & 0.0 & 0.0 & 0.0 & 0.0 & 0.0 & 0.0 & 0.0 & 0.0 & 0.0 & 0.0 & 0.0 \\
\hline
\end{tabular}


TABLE 1 - Continued

\begin{tabular}{|c|c|c|c|c|c|c|c|c|c|c|c|c|c|c|c|c|}
\hline \multicolumn{17}{|c|}{ Hole 62.1 - Continued } \\
\hline Core & $\begin{array}{l}\text { Downhole } \\
\text { Depth of } \\
\text { Core }\end{array}$ & $\begin{array}{c}\text { Depth } \\
\text { Range } \\
\text { Sampled }\end{array}$ & Diff. & $\begin{array}{c}\% \\
\text { Amorphous }\end{array}$ & Calc. & Quar. & Plag. & Kaol. & Mica & Mont. & Clin. & $\mathrm{K}-\mathrm{Fe}$ & Paly. & Barite & Phil. & Crist. \\
\hline 21 & $195-204$ & $\begin{array}{l}195.07- \\
203.21\end{array}$ & 71.1 & 15.0 & 100.0 & 0.0 & 0.0 & 0.0 & 0.0 & 0.0 & 0.0 & 0.0 & 0.0 & 0.0 & 0.0 & 0.0 \\
\hline 22 & $207-216$ & $\begin{array}{l}207.06- \\
215.21\end{array}$ & 71.0 & 14.7 & 100.0 & 0.0 & 0.0 & 0.0 & 0.0 & 0.0 & 0.0 & 0.0 & 0.0 & 0.0 & 0.0 & 0.0 \\
\hline 23 & $216-225$ & $\begin{array}{l}216.10- \\
224.21\end{array}$ & 69.6 & 10.6 & 100.0 & 0.0 & 0.0 & 0.0 & 0.0 & 0.0 & 0.0 & 0.0 & 0.0 & 0.0 & 0.0 & 0.0 \\
\hline 24 & $225-234$ & $\begin{array}{l}225.07- \\
233.21\end{array}$ & 70.3 & 12.6 & 100.0 & 0.0 & 0.0 & 0.0 & 0.0 & 0.0 & 0.0 & 0.0 & 0.0 & 0.0 & 0.0 & 0.0 \\
\hline 25 & $234-243$ & $\begin{array}{l}234.09- \\
242.21\end{array}$ & 70.3 & 12.6 & 100.0 & 0.0 & 0.0 & 0.0 & 0.0 & 0.0 & 0.0 & 0.0 & 0.0 & 0.0 & 0.0 & 0.0 \\
\hline 26 & $245-254$ & $\begin{array}{l}245.10- \\
253.21\end{array}$ & 71.0 & 14.7 & 100.0 & 0.0 & 0.0 & 0.0 & 0.0 & 0.0 & 0.0 & 0.0 & 0.0 & 0.0 & 0.0 & 0.0 \\
\hline 27 & $254-263$ & $\begin{array}{l}255.11- \\
260.71\end{array}$ & 70.8 & 14.1 & 100.0 & 0.0 & 0.0 & 0.0 & 0.0 & 0.0 & 0.0 & 0.0 & 0.0 & 0.0 & 0.0 & 0.0 \\
\hline 28 & $263-271$ & $\begin{array}{l}263.06- \\
271.00\end{array}$ & 70.5 & 13.2 & 100.0 & 0.0 & 0.0 & 0.0 & 0.0 & 0.0 & 0.0 & 0.0 & 0.0 & 0.0 & 0.0 & 0.0 \\
\hline 29 & $271-281$ & $\begin{array}{l}271.06- \\
279.21\end{array}$ & 71.5 & 16.2 & 100.0 & 0.0 & 0.0 & 0.0 & 0.0 & 0.0 & 0.0 & 0.0 & 0.0 & 0.0 & 0.0 & 0.0 \\
\hline 30 & $281 \cdot 291$ & $\begin{array}{l}281.05- \\
289.21\end{array}$ & 71.1 & 15.0 & 100.0 & 0.0 & 0.0 & 0.0 & 0.0 & 0.0 & 0.0 & 0.0 & 0.0 & 0.0 & 0.0 & 0.0 \\
\hline 31 & $291-300$ & $\begin{array}{l}291.02- \\
299.21\end{array}$ & 71.5 & 13.2 & 100.0 & 0.0 & 0.0 & 0.0 & 0.0 & 0.0 & 0.0 & 0.0 & 0.0 & 0.0 & 0.0 & 0.0 \\
\hline 32 & $301-310$ & $\begin{array}{l}301.04- \\
309.21\end{array}$ & 71.4 & 15.9 & 100.0 & 0.0 & 0.0 & 0.0 & 0.0 & 0.0 & 0.0 & 0.0 & 0.0 & 0.0 & 0.0 & 0.0 \\
\hline 33 & $310-319$ & $\begin{array}{l}311.55- \\
313.71\end{array}$ & 71.4 & 15.9 & 100.0 & 0.0 & 0.0 & 0.0 & 0.0 & 0.0 & 0.0 & 0.0 & 0.0 & 0.0 & 0.0 & 0.0 \\
\hline 34 & $320-327^{*}$ & $\begin{array}{l}319.07-* \\
327.99\end{array}$ & 72.2 & 18.2 & 100.0 & 0.0 & 0.0 & 0.0 & 0.0 & 0.0 & 0.0 & 0.0 & 0.0 & 0.0 & 0.0 & 0.0 \\
\hline 35 & $327-336$ & $\begin{array}{l}327.33- \\
335.21\end{array}$ & 71.8 & 17.1 & 100.0 & 0.0 & 0.0 & 0.0 & 0.0 & 0.0 & 0.0 & 0.0 & 0.0 & 0.0 & 0.0 & 0.0 \\
\hline
\end{tabular}


TABLE 1 - Continued

\begin{tabular}{|c|c|c|c|c|c|c|c|c|c|c|c|c|c|c|c|c|}
\hline \multicolumn{17}{|c|}{ Hole 62.1 - Continued } \\
\hline Core & $\begin{array}{l}\text { Downhole } \\
\text { Depth of } \\
\text { Core }\end{array}$ & $\begin{array}{c}\text { Depth } \\
\text { Range } \\
\text { Sampled }\end{array}$ & Diff. & $\begin{array}{c}\% \\
\text { Amorphous }\end{array}$ & Calc. & Quar. & Plag. & Kaol. & Mica & Mont. & Clin. & $\mathrm{K}-\mathrm{Fe}$ & Paly. & Barite & Phil. & Crist. \\
\hline 36 & $336-344$ & $\begin{array}{l}337.55- \\
342.71\end{array}$ & 72.4 & 18.8 & 100.0 & 0.0 & 0.0 & 0.0 & 0.0 & 0.0 & 0.0 & 0.0 & 0.0 & 0.0 & 0.0 & 0.0 \\
\hline 37 & $344-350$ & $\begin{array}{l}345.55- \\
346.21\end{array}$ & 72.5 & 19.1 & 100.0 & 0.0 & 0.0 & 0.0 & 0.0 & 0.0 & 0.0 & 0.0 & 0.0 & 0.0 & 0.0 & 0.0 \\
\hline \multicolumn{17}{|c|}{ Hole 63.0} \\
\hline Core & $\begin{array}{l}\text { Downhole } \\
\text { Depth of } \\
\text { Core }\end{array}$ & $\begin{array}{c}\text { Depth } \\
\text { Range } \\
\text { Sampled }\end{array}$ & Diff. & $\begin{array}{c}\% \\
\text { Amorphous }\end{array}$ & Calc. & Quar. & Plag. & Kaol. & Mica & Mont. & Clin. & $\mathrm{K}-\mathrm{Fe}$ & Paly. & Barite & Phil. & Crist. \\
\hline 1 & $0-9$ & $\begin{array}{l}0.06- \\
8.22\end{array}$ & 92.8 & 78.8 & 13.7 & 14.3 & 6.4 & 10.9 & 16.4 & 38.2 & 0.0 & 0.0 & 0.0 & 0.0 & 0.0 & 0.0 \\
\hline 2 & $61-70$ & $\begin{array}{l}62.55- \\
69.21\end{array}$ & 71.0 & 14.7 & 100.0 & 0.0 & 0.0 & 0.0 & 0.0 & 0.0 & 0.0 & 0.0 & 0.0 & 0.0 & 0.0 & 0.0 \\
\hline 3 & $137-146$ & $\begin{array}{l}137.50- \\
142.21\end{array}$ & 70.5 & 13.2 & 100.0 & 0.0 & 0.0 & 0.0 & 0.0 & 0.0 & 0.0 & 0.0 & 0.0 & 0.0 & 0.0 & 0.0 \\
\hline 4 & $230-240$ & $\begin{array}{l}230.10- \\
232.21\end{array}$ & 69.8 & 11.2 & 100.0 & 0.0 & 0.0 & 0.0 & 0.0 & 0.0 & 0.0 & 0.0 & 0.0 & 0.0 & 0.0 & 0.0 \\
\hline 5 & $352-359$ & $\begin{array}{l}352.14- \\
354.22\end{array}$ & 68.3 & 6.8 & 100.0 & 0.0 & 0.0 & 0.0 & 0.0 & 0.0 & 0.0 & 0.0 & 0.0 & 0.0 & 0.0 & 0.0 \\
\hline 6 & $458-467$ & $\begin{array}{l}458.06- \\
466.21\end{array}$ & 69.0 & 8.8 & 99.0 & 1.0 & 0.0 & 0.0 & 0.0 & 0.0 & 0.0 & 0.0 & 0.0 & 0.0 & 0.0 & 0.0 \\
\hline 7 & $534-543$ & $\begin{array}{l}534.04- \\
542.21\end{array}$ & 69.4 & 10.0 & 99.0 & 1.0 & 0.0 & 0.0 & 0.0 & 0.0 & 0.0 & 0.0 & 0.0 & 0.0 & 0.0 & 0.0 \\
\hline 8 & $543-553$ & $\begin{array}{l}543.52- \\
547.49\end{array}$ & 69.2 & 9.4 & 100.0 & 0.0 & 0.0 & 0.0 & 0.0 & 0.0 & 0.0 & 0.0 & 0.0 & 0.0 & 0.0 & 0.0 \\
\hline 9 & $553-561$ & $\begin{array}{l}553-43- \\
557.64\end{array}$ & 70.6 & 13.5 & 100.0 & 0.0 & 0.0 & 0.0 & 0.0 & 0.0 & 0.0 & 0.0 & 0.0 & 0.0 & 0.0 & 0.0 \\
\hline
\end{tabular}


TABLE 1 - Continued

\begin{tabular}{|c|c|c|c|c|c|c|c|c|c|c|c|c|c|c|c|c|}
\hline \multicolumn{17}{|c|}{ Hole 63.1} \\
\hline Core & $\begin{array}{l}\text { Downhole } \\
\text { Depth of } \\
\text { Core }\end{array}$ & $\begin{array}{c}\text { Depth } \\
\text { Range } \\
\text { Sampled }\end{array}$ & Diff. & $\begin{array}{c}\% \\
\text { Amorphous }\end{array}$ & Calc. & Quar. & Plag. & Kaol. & Mica & Mont. & Clin. & $\mathrm{K}-\mathrm{Fe}$ & Paly. & Barite & Phil. & Crist. \\
\hline 1 & $3-13$ & $\begin{array}{l}3.50- \\
8.10\end{array}$ & 90.5 & 72.1 & 30.0 & 11.4 & 6.2 & 7.5 & 8.8 & 36.1 & 0.0 & 0.0 & 0.0 & 0.0 & 0.0 & 0.0 \\
\hline 3 & $22-32$ & $\begin{array}{l}22.58- \\
24.21\end{array}$ & 86.3 & 59.7 & 65.6 & 5.8 & 2.6 & 5.5 & 8.4 & 12.2 & 0.0 & 0.0 & 0.0 & 0.0 & 0.0 & 0.0 \\
\hline 5 & $101-110$ & $\begin{array}{l}101.55- \\
106.21\end{array}$ & 75.1 & 26.8 & 74.1 & 7.3 & 4.6 & 4.6 & 9.3 & 0.0 & 0.0 & 0.0 & 0.0 & 0.0 & 0.0 & 0.0 \\
\hline 6 & $110-119$ & $\begin{array}{l}110.65- \\
118.22\end{array}$ & 81.3 & 45.0 & 96.0 & 2.5 & 1.5 & 0.0 & 0.0 & 0.0 & 0.0 & 0.0 & 0.0 & 0.0 & 0.0 & 0.0 \\
\hline 7 & $119-129$ & $\begin{array}{l}119.05- \\
122.71\end{array}$ & 78.9 & 37.9 & 98.5 & 1.5 & 0.0 & 0.0 & 0.0 & 0.0 & 0.0 & 0.0 & 0.0 & 0.0 & 0.0 & 0.0 \\
\hline 8 & $129-138$ & $\begin{array}{l}130.63- \\
137.21\end{array}$ & 75.5 & 27.9 & 99.0 & 1.0 & 0.0 & 0.0 & 0.0 & 0.0 & 0.0 & 0.0 & 0.0 & 0.0 & 0.0 & 0.0 \\
\hline \multirow[t]{2}{*}{9} & $138-148$ & $\begin{array}{l}138.12- \\
146.21\end{array}$ & 73.1 & 20.9 & 99.0 & 1.0 & 0.0 & 0.0 & 0.0 & 0.0 & 0.0 & 0.0 & 0.0 & 0.0 & 0.0 & 0.0 \\
\hline & & $\begin{array}{l}143.24- \\
143.26\end{array}$ & 69.2 & 9.4 & 100.0 & 0.0 & 0.0 & 0.0 & 0.0 & 0.0 & 0.0 & 0.0 & 0.0 & 0.0 & 0.0 & 0.0 \\
\hline 10 & $148-155$ & $\begin{array}{l}148.16- \\
154.76\end{array}$ & 72.5 & 19.1 & 100.0 & 0.0 & 0.0 & 0.0 & 0.0 & 0.0 & 0.0 & 0.0 & 0.0 & 0.0 & 0.0 & 0.0 \\
\hline 11 & $155-165$ & $\begin{array}{l}155.03- \\
163.21\end{array}$ & 71.9 & 17.4 & 100.0 & 0.0 & 0.0 & 0.0 & 0.0 & 0.0 & 0.0 & 0.0 & 0.0 & 0.0 & 0.0 & 0.0 \\
\hline 12 & $165-174$ & $\begin{array}{l}166.62- \\
167.21\end{array}$ & 70.7 & 13.8 & 100.0 & 0.0 & 0.0 & 0.0 & 0.0 & 0.0 & 0.0 & 0.0 & 0.0 & 0.0 & 0.0 & 0.0 \\
\hline 13 & $174-184$ & $\begin{array}{l}174.05- \\
182.00\end{array}$ & 74.1 & 23.8 & 99.0 & 1.0 & 0.0 & 0.0 & 0.0 & 0.0 & 0.0 & 0.0 & 0.0 & 0.0 & 0.0 & 0.0 \\
\hline 14 & $184-193$ & $\begin{array}{l}184.06- \\
192.33\end{array}$ & 72.3 & 18.5 & 99.0 & 1.0 & 0.0 & 0.0 & 0.0 & 0.0 & 0.0 & 0.0 & 0.0 & 0.0 & 0.0 & 0.0 \\
\hline
\end{tabular}


TABLE 1 - Continued

\begin{tabular}{|c|c|c|c|c|c|c|c|c|c|c|c|c|c|c|c|c|}
\hline \multicolumn{17}{|c|}{ Hole 63.2} \\
\hline Core & $\begin{array}{l}\text { Downhole } \\
\text { Depth of } \\
\text { Core }\end{array}$ & $\begin{array}{c}\text { Depth } \\
\text { Range } \\
\text { Sampled }\end{array}$ & Diff. & $\begin{array}{c}\% \\
\text { Amorphous }\end{array}$ & Calc. & Quar. & Plag. & Kaol. & Mica & Mont. & Clin. & $\mathrm{K}-\mathrm{Fe}$ & Paly. & Barite & Phil & Crist. \\
\hline 1 & $11-20$ & $\begin{array}{l}11.05- \\
16.21\end{array}$ & 90.2 & 71.2 & 52.7 & 12.4 & 6.3 & 11.0 & 17.6 & 0.0 & 0.0 & 0.0 & 0.0 & 0.0 & 0.0 & 0.0 \\
\hline 2 & $20-30$ & $\begin{array}{l}20.05- \\
28.67\end{array}$ & 88.3 & 65.6 & 67.6 & 11.6 & 5.3 & 7.0 & 8.5 & 0.0 & 0.0 & 0.0 & 0.0 & 0.0 & 0.0 & 0.0 \\
\hline 3 & $30-89$ & $\begin{array}{l}30.42- \\
35.21\end{array}$ & 82.2 & 47.6 & 86.2 & 4.5 & 2.0 & 3.6 & 3.7 & 0.0 & 0.0 & 0.0 & 0.0 & 0.0 & 0.0 & 0.0 \\
\hline \multicolumn{17}{|c|}{ Hole 64.0} \\
\hline Core & $\begin{array}{l}\text { Downhole } \\
\text { Depth of } \\
\text { Core }\end{array}$ & $\begin{array}{c}\text { Depth } \\
\text { Range } \\
\text { Sampled }\end{array}$ & Diff. & $\begin{array}{c}\% \\
\text { Amorphous }\end{array}$ & Calc. & Quar. & Plag. & Kaol. & Mica & Mont. & Clin. & $\mathrm{K}-\mathrm{Fe}$ & Paly & Barite & Phil. & Crist. \\
\hline 1 & $0-9$ & $\begin{array}{l}0.04- \\
8.51\end{array}$ & 74.8 & 25.9 & 97.5 & 1.0 & 1.5 & 0.0 & 0.0 & 0.0 & 0.0 & 0.0 & 0.0 & 0.0 & 0.0 & 0.0 \\
\hline 2 & $99-108$ & $\begin{array}{r}99.37- \\
107.21\end{array}$ & 70.1 & 12.1 & 100.0 & 0.0 & 0.0 & 0.0 & 0.0 & 0.0 & 0.0 & 0.0 & 0.0 & 0.0 & 0.0 & 0.0 \\
\hline 3 & $202-211$ & $\begin{array}{l}202.04- \\
210.21\end{array}$ & 68.4 & 7.1 & 100.0 & 0.0 & 0.0 & 0.0 & 0.0 & 0.0 & 0.0 & 0.0 & 0.0 & 0.0 & 0.0 & 0.0 \\
\hline 4 & $304-313$ & $\begin{array}{l}304.51- \\
312.21\end{array}$ & 68.5 & 7.4 & 100.0 & 0.0 & 0.0 & 0.0 & 0.0 & 0.0 & 0.0 & 0.0 & 0.0 & 0.0 & 0.0 & 0.0 \\
\hline 5 & $409-418$ & $\begin{array}{l}409.11- \\
417.29\end{array}$ & 69.1 & 9.1 & 100.0 & 0.0 & 0.0 & 0.0 & 0.0 & 0.0 & 0.0 & 0.0 & 0.0 & 0.0 & 0.0 & 0.0 \\
\hline 6 & $505-514$ & $\begin{array}{l}505.08- \\
513.22\end{array}$ & 69.2 & 9.4 & 100.0 & 0.0 & 0.0 & 0.0 & 0.0 & 0.0 & 0.0 & 0.0 & 0.0 & 0.0 & 0.0 & 0.0 \\
\hline 7 & $610-619$ & $\begin{array}{l}610.25- \\
618.22\end{array}$ & 68.9 & 8.5 & 100.0 & 0.0 & 0.0 & 0.0 & 0.0 & 0.0 & 0.0 & 0.0 & 0.0 & 0.0 & 0.0 & 0.0 \\
\hline 8 & $705-710$ & $\begin{array}{l}705.37- \\
708.71\end{array}$ & 70.7 & 13.8 & 100.0 & 0.0 & 0.0 & 0.0 & 0.0 & 0.0 & 0.0 & 0.0 & 0.0 & 0.0 & 0.0 & 0.0 \\
\hline 10 & $848-851$ & $\begin{array}{l}848.82- \\
850.21\end{array}$ & 69.5 & 10.3 & 100.0 & 0.0 & 0.0 & 0.0 & 0.0 & 0.0 & 0.0 & 0.0 & 0.0 & 0.0 & 0.0 & 0.0 \\
\hline
\end{tabular}




\begin{tabular}{|c|c|c|c|c|c|c|c|c|c|c|c|c|c|c|c|c|}
\hline \multicolumn{17}{|c|}{ Hole 64.1} \\
\hline Core & $\begin{array}{l}\text { Downhole } \\
\text { Depth of } \\
\text { Core }\end{array}$ & $\begin{array}{c}\text { Depth } \\
\text { Range } \\
\text { Sampled }\end{array}$ & Diff. & $\begin{array}{c}\% \\
\text { Amorphous }\end{array}$ & Calc. & Quar. & Plag. & Kaol. & Mica & Mont. & Clin. & $\mathrm{K} \cdot \mathrm{Fe}$ & Paly. & Barite & Phil. & Crist. \\
\hline 1 & $433-442$ & $\begin{array}{l}433.13- \\
441.22\end{array}$ & 68.6 & 7.6 & 100.0 & 0.0 & 0.0 & 0.0 & 0.0 & 0.0 & 0.0 & 0.0 & 0.0 & 0.0 & 0.0 & 0.0 \\
\hline 2 & $442-451$ & $\begin{array}{l}442.30- \\
450.21\end{array}$ & 69.0 & 8.8 & 100.0 & 0.0 & 0.0 & 0.0 & 0.0 & 0.0 & 0.0 & 0.0 & 0.0 & 0.0 & 0.0 & 0.0 \\
\hline 3 & $451-461$ & $\begin{array}{l}451.13- \\
459.19\end{array}$ & 68.9 & 8.5 & 100.0 & 0.0 & 0.0 & 0.0 & 0.0 & 0.0 & 0.0 & 0.0 & 0.0 & 0.0 & 0.0 & 0.0 \\
\hline 4 & $461-470$ & $\begin{array}{l}461.13- \\
469.21\end{array}$ & 70.4 & 12.9 & 100.0 & 0.0 & 0.0 & 0.0 & 0.0 & 0.0 & 0.0 & 0.0 & 0.0 & 0.0 & 0.0 & 0.0 \\
\hline 5 & $470-479$ & $\begin{array}{l}470.29- \\
478.21\end{array}$ & 69.3 & 9.7 & 100.0 & 0.0 & 0.0 & 0.0 & 0.0 & 0.0 & 0.0 & 0.0 & 0.0 & 0.0 & 0.0 & 0.0 \\
\hline 6 & $565-571$ & $\begin{array}{l}565.06- \\
570.31\end{array}$ & 67.5 & 4.4 & 100.0 & 0.0 & 0.0 & 0.0 & 0.0 & 0.0 & 0.0 & 0.0 & 0.0 & 0.0 & 0.0 & 0.0 \\
\hline 7 & $661-663^{*}$ & $\begin{array}{l}662.03 . \\
666.22^{*}\end{array}$ & 71.1 & 15.0 & 100.0 & 0.0 & 0.0 & 0.0 & 0.0 & 0.0 & 0.0 & 0.0 & 0.0 & 0.0 & 0.0 & 0.0 \\
\hline 8 & $746-749$ & $\begin{array}{l}747.03- \\
748.21\end{array}$ & 68.1 & 6.2 & 100.0 & 0.0 & 0.0 & 0.0 & 0.0 & 0.0 & 0.0 & 0.0 & 0.0 & 0.0 & 0.0 & 0.0 \\
\hline 9 & $911-916$ & $\begin{array}{l}911.12- \\
914.72\end{array}$ & 68.9 & 8.5 & 100.0 & 0.0 & 0.0 & 0.0 & 0.0 & 0.0 & 0.0 & 0.0 & 0.0 & 0.0 & 0.0 & 0.0 \\
\hline 10 & $969-972$ & $\begin{array}{l}969.16- \\
971.21\end{array}$ & 69.6 & 10.6 & 100.0 & 0.0 & 0.0 & 0.0 & 0.0 & 0.0 & 0.0 & 0.0 & 0.0 & 0.0 & 0.0 & 0.0 \\
\hline \multicolumn{17}{|c|}{ Hole 65.0} \\
\hline Core & $\begin{array}{l}\text { Downhole } \\
\text { Depth of } \\
\text { Core }\end{array}$ & $\begin{array}{c}\text { Depth } \\
\text { Range } \\
\text { Sampled }\end{array}$ & Diff. & $\begin{array}{c}\% \\
\text { Amorphous }\end{array}$ & Calc. & Quar. & Plag. & Kaol. & Mica & Mont. & Clin. & $\mathrm{K}-\mathrm{Fe}$ & Paly. & Barite & Phil. & Crist. \\
\hline 2 & $10-19$ & $\begin{array}{l}10.42- \\
16.91\end{array}$ & 95.2 & 85.9 & 0.0 & 10.9 & 7.1 & 0.0 & 14.6 & 67.4 & 0.0 & 0.0 & 0.0 & 0.0 & 0.0 & 0.0 \\
\hline 3 & $19-28$ & $\begin{array}{l}19.19- \\
25.71\end{array}$ & 97.2 & 91.8 & 0.0 & 30.9 & 19.6 & 0.0 & 49.5 & 0.0 & 0.0 & 0.0 & 0.0 & 0.0 & 0.0 & 0.0 \\
\hline
\end{tabular}


TABLE 1 - Continued

\begin{tabular}{|c|c|c|c|c|c|c|c|c|c|c|c|c|c|c|c|c|}
\hline \multicolumn{17}{|c|}{ Hole 65.0 - Continued } \\
\hline Core & $\begin{array}{l}\text { Downhole } \\
\text { Depth of } \\
\text { Core }\end{array}$ & $\begin{array}{c}\text { Depth } \\
\text { Range } \\
\text { Sampled }\end{array}$ & Diff. & $\begin{array}{c}\% \\
\text { Amorphous }\end{array}$ & Calc. & Quar. & Plag. & Kaol. & Mica & Mont. & Clin. & $\mathrm{K}-\mathrm{Fe}$ & Paly. & Barite & Phil. & Crist. \\
\hline 4 & $28-37$ & $\begin{array}{l}29.65- \\
34.71\end{array}$ & 96.7 & 90.3 & 0.0 & 58.7 & 41.3 & 0.0 & 0.0 & 0.0 & 0.0 & 0.0 & 0.0 & 0.0 & 0.0 & 0.0 \\
\hline 5 & $37-46$ & $\begin{array}{l}38.92- \\
45.21\end{array}$ & 97.4 & 92.4 & 0.0 & 21.0 & 21.7 & 0.0 & 57.3 & 0.0 & 0.0 & 0.0 & 0.0 & 0.0 & 0.0 & 0.0 \\
\hline 6 & $46-55$ & $\begin{array}{l}47.94- \\
48.92\end{array}$ & 98.9 & 96.8 & 0.0 & 54.4 & 45.6 & 0.0 & 0.0 & 0.0 & 0.0 & 0.0 & 0.0 & 0.0 & 0.0 & 0.0 \\
\hline 7 & $55-64$ & $\begin{array}{l}56.89- \\
61.92\end{array}$ & 96.8 & 90.6 & 0.0 & 56.9 & 43.1 & 0.0 & 0.0 & 0.0 & 0.0 & 0.0 & 0.0 & 0.0 & 0.0 & 0.0 \\
\hline 8 & $64-74$ & $\begin{array}{l}64.10- \\
72.21\end{array}$ & 97.5 & 92.6 & 0.0 & 72.9 & 27.1 & 0.0 & 0.0 & 0.0 & 0.0 & 0.0 & 0.0 & 0.0 & 0.0 & 0.0 \\
\hline 9 & $74-83$ & $\begin{array}{l}74.24- \\
82.41\end{array}$ & 96.9 & 90.9 & 0.0 & 64.2 & 35.8 & 0.0 & 0.0 & 0.0 & 0.0 & 0.0 & 0.0 & 0.0 & 0.0 & 0.0 \\
\hline 10 & $83-92$ & $\begin{array}{l}83.73- \\
88.21\end{array}$ & 96.2 & 88.8 & 0.0 & 57.4 & 42.6 & 0.0 & 0.0 & 0.0 & 0.0 & 0.0 & 0.0 & 0.0 & 0.0 & 0.0 \\
\hline 11 & $92-101$ & $\begin{array}{l}94.18- \\
100.52\end{array}$ & 97.8 & 93.5 & 0.0 & 43.4 & 56.6 & 0.0 & 0.0 & 0.0 & 0.0 & 0.0 & 0.0 & 0.0 & 0.0 & 0.0 \\
\hline 12 & $101-110$ & $\begin{array}{l}101.07- \\
118.41\end{array}$ & 95.4 & 86.5 & 0.0 & 17.2 & 23.3 & 0.0 & 0.0 & 0.0 & 59.5 & 0.0 & 0.0 & 0.0 & 0.0 & 0.0 \\
\hline 13 & $110-119$ & $\begin{array}{l}110.34- \\
118.41\end{array}$ & 97.8 & 93.5 & 0.0 & 33.6 & 66.4 & 0.0 & 0.0 & 0.0 & 0.0 & 0.0 & 0.0 & 0.0 & 0.0 & 0.0 \\
\hline 14 & $119-127 *$ & $\begin{array}{l}119.06- \\
127.21^{*}\end{array}$ & 93.4 & 80.6 & 96.0 & 2.5 & 1.5 & 0.0 & 0.0 & 0.0 & 0.0 & 0.0 & 0.0 & 0.0 & 0.0 & 0.0 \\
\hline 16 & $137-145^{*}$ & $\begin{array}{l}138.97 \\
145.21^{*}\end{array}$ & 94.5 & 83.8 & 89.3 & 5.1 & 5.6 & 0.0 & 0.0 & 0.0 & 0.0 & 0.0 & 0.0 & 0.0 & 0.0 & 0.0 \\
\hline
\end{tabular}




\begin{tabular}{|c|c|c|c|c|c|c|c|c|c|c|c|c|c|c|c|c|}
\hline \multicolumn{17}{|c|}{ Hole 65.1} \\
\hline Core & $\begin{array}{l}\text { Downhole } \\
\text { Depth of } \\
\text { Core }\end{array}$ & $\begin{array}{c}\text { Depth } \\
\text { Range } \\
\text { Sampled }\end{array}$ & Diff. & $\begin{array}{c}\% \\
\text { Amorphous }\end{array}$ & Calc. & Quar. & Plag. & Kaol. & Mica & Mont. & Clin. & $\mathrm{K} \cdot \mathrm{Fe}$ & Paly. & Barite & Phil. & Crist. \\
\hline 2 & $145-147$ & $\begin{array}{l}146.01- \\
146.35\end{array}$ & 86.3 & 59.7 & 70.6 & 1.0 & 1.0 & 0.0 & 0.0 & 20.7 & 4.6 & 0.0 & 0.0 & 0.0 & 0.0 & 0.0 \\
\hline 4 & $154-162 *$ & $\begin{array}{l}153.37- \\
161.21^{*}\end{array}$ & 97.2 & 91.8 & 0.0 & 5.7 & 0.0 & 0.0 & 0.0 & 94.3 & 0.0 & 0.0 & 0.0 & 0.0 & 0.0 & 0.0 \\
\hline 5 & $162-168$ & $\begin{array}{l}162.61- \\
167.88\end{array}$ & 99.1 & 97.4 & 0.0 & 0.0 & 0.0 & 0.0 & 0.0 & 0.0 & 100.0 & 0.0 & 0.0 & 0.0 & 0.0 & 0.0 \\
\hline \multicolumn{17}{|c|}{ Hole 66.0} \\
\hline Core & $\begin{array}{c}\text { Downhole } \\
\text { Depth of } \\
\text { Core }\end{array}$ & $\begin{array}{c}\text { Depth } \\
\text { Range } \\
\text { Sampled }\end{array}$ & Diff. & Amorphous & Calc. & Quar. & Plag. & Kaol. & Mica & Mont. & Clin. & $\mathrm{K} \cdot \mathrm{Fe}$ & Paly. & Barite & Phil. & Crist. \\
\hline 2 & $79-88$ & $\begin{array}{l}79.05- \\
82.73\end{array}$ & 98.1 & 94.4 & 88.1 & 11.9 & 0.0 & 0.0 & 0.0 & 0.0 & 0.0 & 0.0 & 0.0 & 0.0 & 0.0 & 0.0 \\
\hline 3 & $117-126$ & $\begin{array}{l}117.54- \\
125.41\end{array}$ & 97.6 & 99.2 & 0.0 & 19.4 & 0.0 & 0.0 & 0.0 & 0.0 & 80.6 & 0.0 & 0.0 & 0.0 & 0.0 & 0.0 \\
\hline 6 & $165-174$ & $\begin{array}{l}165.09- \\
170.21\end{array}$ & 89.4 & 68.8 & 0.0 & 16.6 & 0.0 & 0.0 & 30.7 & 14.3 & 0.0 & 10.4 & 28.0 & 0.0 & 0.0 & 0.0 \\
\hline 7 & $174-180$ & $\begin{array}{l}174.33- \\
179.21\end{array}$ & 89.3 & 68.5 & 0.0 & 15.8 & 0.0 & 2.0 & 29.4 & 15.0 & 0.0 & 11.1 & 26.7 & 0.0 & 0.0 & 0.0 \\
\hline 8 & $180-187$ & $\begin{array}{l}180.12- \\
186.29\end{array}$ & 89.3 & 68.5 & 0.0 & 15.9 & 0.0 & 3.0 & 29.6 & 13.8 & 0.0 & 10.8 & 26.9 & 0.0 & 0.0 & 0.0 \\
\hline 9 & $187-192$ & $\begin{array}{l}187.69- \\
191.27\end{array}$ & 93.8 & 81.8 & 0.0 & 11.0 & 0.0 & 0.0 & 23.7 & 36.2 & 0.0 & 10.2 & 18.9 & 0.0 & 0.0 & 0.0 \\
\hline
\end{tabular}


TABLE 1 - Continued

\begin{tabular}{|c|c|c|c|c|c|c|c|c|c|c|c|c|c|c|c|c|}
\hline \multicolumn{17}{|c|}{ Hole 66.1} \\
\hline Core & $\begin{array}{l}\text { Downhole } \\
\text { Depth of } \\
\text { Core }\end{array}$ & $\begin{array}{c}\text { Depth } \\
\text { Range } \\
\text { Sampled }\end{array}$ & Diff. & $\begin{array}{c}\% \\
\text { Amorphous }\end{array}$ & Calc. & Quar. & Plag. & Kaol. & Mica & Mont. & Clin. & $\mathrm{K}-\mathrm{Fe}$ & Paly. & Barite & Phil. & Crist. \\
\hline 2 & $20-29$ & $\begin{array}{l}20.11- \\
28.21\end{array}$ & 96.2 & 88.8 & 0.0 & 12.4 & 8.0 & 5.4 & 10.9 & 56.9 & 0.0 & 5.4 & 0.0 & 0.0 & 0.0 & 0.0 \\
\hline 3 & $29-38$ & $\begin{array}{l}29.26- \\
37.19\end{array}$ & 97.6 & 92.9 & 0.0 & 57.9 & 42.1 & 0.0 & 0.0 & 0.0 & 0.0 & 0.0 & 0.0 & 0.0 & 0.0 & 0.0 \\
\hline 4 & $38-47$ & $\begin{array}{l}39.04- \\
46.42\end{array}$ & 97.9 & 93.8 & 0.0 & 15.8 & 0.0 & 0.0 & 0.0 & 84.2 & 0.0 & 0.0 & 0.0 & 0.0 & 0.0 & 0.0 \\
\hline 5 & $47-56$ & $\begin{array}{l}47.06- \\
55.31\end{array}$ & 97.5 & 92.6 & 0.0 & 11.1 & 0.0 & 0.0 & 0.0 & 88.9 & 0.0 & 0.0 & 0.0 & 0.0 & 0.0 & 0.0 \\
\hline 6 & $56-65$ & $\begin{array}{l}57.65- \\
64.32\end{array}$ & 98.2 & 94.7 & 0.0 & 100.0 & 0.0 & 0.0 & 0.0 & 0.0 & 0.0 & 0.0 & 0.0 & 0.0 & 0.0 & 0.0 \\
\hline 7 & $67-76$ & $\begin{array}{l}67.14- \\
75.32\end{array}$ & 98.4 & 95.3 & 0.0 & 100.0 & 0.0 & 0.0 & 0.0 & 0.0 & 0.0 & 0.0 & 0.0 & 0.0 & 0.0 & 0.0 \\
\hline 8 & $76-86$ & $\begin{array}{l}76.05- \\
84.31\end{array}$ & 97.9 & 93.8 & 0.0 & 10.0 & 0.0 & 0.0 & 0.0 & 0.0 & 0.0 & 0.0 & 0.0 & 10.0 & 0.0 & 0.0 \\
\hline
\end{tabular}

\section{Hole 67.0}

\begin{tabular}{cccccccccccccccccccccc}
\hline & $\begin{array}{c}\text { Downhole } \\
\text { Depth of } \\
\text { Core }\end{array}$ & $\begin{array}{c}\text { Depth } \\
\text { Range } \\
\text { Sampled }\end{array}$ & Diff. & Amorphous & Calc. & Quar. & Plag. & Kaol. & Mica & Mont. & Clin. & K-Fe & Paly. & Barite & Phil. & Crist. \\
\hline 1 & $0-5$ & $0.31-1.09$ & 91.3 & 74.4 & 0.0 & 9.7 & 15.5 & 0.0 & 0.0 & 0.0 & 0.0 & 0.0 & 0.0 & 0.0 & 74.8 & 0.0 \\
\hline
\end{tabular}




\begin{tabular}{|c|c|c|c|c|c|c|c|c|c|c|c|c|c|c|c|c|}
\hline \multicolumn{17}{|c|}{ Hole 67.1} \\
\hline Core & $\begin{array}{l}\text { Downhole } \\
\text { Depth of } \\
\text { Core }\end{array}$ & $\begin{array}{c}\text { Depth } \\
\text { Range } \\
\text { Sampled }\end{array}$ & Diff. & $\begin{array}{c}\% \\
\text { Amorphous }\end{array}$ & Calc. & Quar. & Plag. & Kaol. & Mica & Mont. & Clin. & $\mathrm{K}-\mathrm{Fe}$ & Paly. & Barite & Phil. & Crist. \\
\hline \multirow[t]{2}{*}{1} & $23-32$ & $\begin{array}{l}23.65- \\
23.67\end{array}$ & 92.1 & 76.8 & 0.0 & 0.0 & 0.0 & 0.0 & 0.0 & 0.0 & 0.0 & 0.0 & 0.0 & 0.0 & 100.0 & 0.0 \\
\hline & & $\begin{array}{r}\approx 24.50- \\
24.55\end{array}$ & 91.2 & 74.1 & 0.0 & 0.0 & 5.0 & 0.0 & 0.0 & 0.0 & 0.0 & 0.0 & 0.0 & 0.0 & 95.0 & 0.0 \\
\hline
\end{tabular}

Note: Entries of zero indicate that the specific mineral was not present in traceable quantities.

${ }^{\mathrm{a}}$ Abbreviations for X-ray mineralogy results: Calc.-calcite; Quar.-quartz; Plag.-plagioclase; Kaol.-kaolinite; Mont.-montmorillonite;Clin.-clinoptilolite; K-Fe-K-feldspar; Paly.-palygorskite; Phil.-phillipsite; Crist.-cristobalite.

*Depth on plot changed in order to accommodate samples received. 
TABLE 2

X-Ray Diffraction Analysis of Samples from Leg 7: Composited $<2 \mu$ Fraction

\begin{tabular}{|c|c|c|c|c|c|c|c|c|c|c|c|c|c|c|c|c|c|c|}
\hline \multicolumn{19}{|c|}{ Hole 61.1} \\
\hline Core & $\begin{array}{l}\text { Downhole } \\
\text { Depth of } \\
\text { Core }\end{array}$ & $\begin{array}{c}\text { Depth } \\
\text { Range } \\
\text { Sampled }\end{array}$ & Diff. & $\begin{array}{c}\% \\
\text { Amorphous }\end{array}$ & Quar. & Plag. & Kaol. & Mica & Mont. & Crist. & Clin. & $\mathrm{K} \cdot \mathrm{Fe}$ & Pyrite & Barite & Apat. & Side. & Paly. & Phil. \\
\hline 1 & $83-89$ & $\begin{array}{l}84.04- \\
85.82\end{array}$ & 68.8 & 51.2 & 4.7 & 3.1 & 0.0 & 0.0 & 36.5 & 55.7 & 0.0 & 0.0 & 0.0 & 0.0 & 0.0 & 0.0 & 0.0 & 0.0 \\
\hline \multicolumn{19}{|c|}{ Hole 62.0} \\
\hline Core & $\begin{array}{l}\text { Downhole } \\
\text { Depth of } \\
\text { Core }\end{array}$ & $\begin{array}{c}\text { Depth } \\
\text { Range } \\
\text { Sampled }\end{array}$ & Diff. & $\begin{array}{c}\% \\
\text { Amorphous }\end{array}$ & Quar. & Plag. & Kaol. & Mica & Mont. & Crist. & Clin. & $\mathrm{K}-\mathrm{Fe}$ & Pyrite & Barite & Apat. & Side. & Paly. & Phil. \\
\hline 2 & $205-214$ & $\begin{array}{l}205.89- \\
213.69\end{array}$ & 88.3 & 81.7 & 6.6 & 5.6 & 8.3 & 16.2 & 63.2 & 0.0 & 0.0 & 0.0 & 0.0 & 0.0 & 0.0 & 0.0 & 0.0 & 0.0 \\
\hline 3 & $299-308$ & $\begin{array}{l}299.04- \\
307.21\end{array}$ & 87.5 & 80.5 & 9.0 & 7.1 & 15.2 & 30.8 & 32.9 & 0.0 & 0.0 & 2.8 & 2.3 & 0.0 & 0.0 & 0.0 & 0.0 & 0.0 \\
\hline 4 & $395-404$ & $\begin{array}{l}395.08- \\
403.23\end{array}$ & 86.3 & 78.6 & 10.9 & 4.0 & 5.1 & 25.7 & 42.4 & 0.0 & 11.6 & 0.0 & 0.0 & 0.0 & 0.0 & 0.0 & 0.0 & 0.0 \\
\hline 5 & $490-496$ & $\begin{array}{l}491.67- \\
495.23\end{array}$ & 88.6 & 82.2 & 4.3 & 3.2 & 5.0 & 17.0 & 70.4 & 0.0 & 0.0 & 0.0 & 0.0 & 0.0 & 0.0 & 0.0 & 0.0 & 0.0 \\
\hline \multicolumn{19}{|c|}{ Hole 62.1} \\
\hline Core & $\begin{array}{l}\text { Downhole } \\
\text { Depth of } \\
\text { Core }\end{array}$ & $\begin{array}{l}\text { Depth } \\
\text { Range } \\
\text { Sampled }\end{array}$ & Diff. & $\begin{array}{c}\% \\
\text { Amorphous }\end{array}$ & Quar. & Plag. & Kaol. & Mica & Mont. & Crist. & Clin. & $\mathrm{K}-\mathrm{Fe}$ & Pyrite & Barite & Apat. & Side & Paly. & Phil. \\
\hline 1 & $6-15$ & $\begin{array}{l}6.33- \\
8.23\end{array}$ & 86.6 & 79.1 & 12.2 & 10.5 & 3.3 & 45.4 & 28.7 & 0.0 & 0.0 & 0.0 & 0.0 & 0.0 & 0.0 & 0.0 & 0.0 & 0.0 \\
\hline 2 & $15-24$ & $\begin{array}{l}16.55= \\
20.25\end{array}$ & 92.3 & 77.4 & 5.7 & 2.4 & 6.1 & 5.1 & 80.7 & 0.0 & 0.0 & 0.0 & 0.0 & 0.0 & 0.0 & 0.0 & 0.0 & 0.0 \\
\hline 4 & $34-43$ & $\begin{array}{l}34.05- \\
42.21\end{array}$ & 92.9 & 79.1 & 4.9 & 2.2 & 5.3 & 0.0 & 87.6 & 0.0 & 0.0 & 0.0 & 0.0 & 0.0 & 0.0 & 0.0 & 0.0 & 0.0 \\
\hline 6 & $54-63$ & $\begin{array}{l}54.13- \\
62.21\end{array}$ & 92.4 & 77.6 & 4.8 & 2.8 & 5.4 & 5.5 & 81.4 & 0.0 & 0.0 & 0.0 & 0.0 & 0.0 & 0.0 & 0.0 & 0.0 & 0.0 \\
\hline
\end{tabular}




\begin{tabular}{|c|c|c|c|c|c|c|c|c|c|c|c|c|c|c|c|c|c|c|}
\hline \multicolumn{19}{|c|}{ Hole 62.1 - Continued } \\
\hline Core & $\begin{array}{l}\text { Downhole } \\
\text { Depth of } \\
\text { Core }\end{array}$ & $\begin{array}{c}\text { Depth } \\
\text { Range } \\
\text { Sampled }\end{array}$ & Diff. & $\begin{array}{c}\% \\
\text { Amorphous }\end{array}$ & Quar. & Plag. & Kaol. & Mica & Mont. & Crist. & Clin. & $\mathrm{K}-\mathrm{Fe}$ & Pyrite & Barite & Apat. & Side. & Paly. & Phil. \\
\hline 7 & $63-72$ & $\begin{array}{l}63.05- \\
71.21\end{array}$ & 88.3 & 81.7 & 12.7 & 12.0 & 3.4 & 35.6 & 32.7 & 0.0 & 0.0 & 0.0 & 3.7 & 0.0 & 0.0 & 0.0 & 0.0 & 0.0 \\
\hline 9 & $81-91$ & $\begin{array}{l}82.55- \\
86.21\end{array}$ & 88.6 & 82.2 & 12.6 & 11.3 & 10.5 & 44.1 & 21.5 & 0.0 & 0.0 & 0.0 & 0.0 & 0.0 & 0.0 & 0.0 & 0.0 & 0.0 \\
\hline 11 & $101-110$ & $\begin{array}{l}101.05- \\
109.48\end{array}$ & 88.3 & 81.7 & 10.2 & 7.8 & 10.2 & 35.6 & 36.2 & 0.0 & 0.0 & 0.0 & 0.0 & 0.0 & 0.0 & 0.0 & 0.0 & 0.0 \\
\hline 12 & $110-119$ & $\begin{array}{l}110.06- \\
118.21\end{array}$ & 86.9 & 79.5 & 10.5 & 10.8 & 15.6 & 31.2 & 22.2 & 0.0 & 0.0 & 0.0 & 9.7 & 0.0 & 0.0 & 0.0 & 0.0 & 0.0 \\
\hline 13 & $119-129$ & $\begin{array}{l}122.94 \\
127.55\end{array}$ & 84.3 & 75.5 & 7.4 & 7.5 & 11.0 & 16.4 & 56.5 & 0.0 & 0.0 & 0.0 & 1.3 & 0.0 & 0.0 & 0.0 & 0.0 & 0.0 \\
\hline 14 & $129-138$ & $\begin{array}{l}129.55- \\
137.66\end{array}$ & 85.8 & 77.8 & 11.4 & 11.0 & 13.2 & 25.4 & 20.6 & 0.0 & 0.0 & 0.0 & 18.4 & 0.0 & 0.0 & 0.0 & 0.0 & 0.0 \\
\hline 15 & $138-147$ & $\begin{array}{l}138.05- \\
146.21\end{array}$ & 88.4 & 81.9 & 11.8 & 11.9 & 14.7 & 35.1 & 23.9 & 0.0 & 0.0 & 0.0 & 2.6 & 0.0 & 0.0 & 0.0 & 0.0 & 0.0 \\
\hline 16 & $147-156$ & $\begin{array}{l}147.06- \\
155.21\end{array}$ & 88.7 & 82.3 & 7.8 & 7.9 & 14.1 & 30.7 & 38.5 & 0.0 & 0.0 & 0.0 & 1.0 & 0.0 & 0.0 & 0.0 & 0.0 & 0.0 \\
\hline 17 & $156-165$ & $\begin{array}{l}157.26- \\
164.18\end{array}$ & 87.5 & 80.5 & 10.2 & 9.2 & 13.5 & 33.2 & 32.8 & 0.0 & 0.0 & 0.0 & 1.1 & 0.0 & 0.0 & 0.0 & 0.0 & 0.0 \\
\hline 18 & $165-174$ & $\begin{array}{l}165.05- \\
173.19\end{array}$ & 87.2 & 80.0 & 9.6 & 7.7 & 14.9 & 34.1 & 33.7 & 0.0 & 0.0 & 0.0 & 0.0 & 0.0 & 0.0 & 0.0 & 0.0 & 0.0 \\
\hline 19 & $174-183$ & $\begin{array}{l}174.82- \\
182.21\end{array}$ & 88.4 & 81.9 & 9.1 & 6.5 & 15.9 & 30.4 & 36.7 & 0.0 & 0.0 & 0.0 & 1.3 & 0.0 & 0.0 & 0.0 & 0.0 & 0.0 \\
\hline 20 & $186-195$ & $\begin{array}{l}186.13- \\
194.21\end{array}$ & 86.8 & 79.4 & 6.7 & 5.0 & 7.4 & 12.3 & 68.4 & 0.0 & 0.0 & 0.0 & 0.0 & 0.0 & 0.0 & 0.0 & 0.0 & 0.0 \\
\hline 21 & $195-204$ & $\begin{array}{l}195.07- \\
203.21\end{array}$ & 87.2 & $80.0^{\circ}$ & 8.2 & 6.0 & 9.6 & 12.8 & 59.8 & 0.0 & 0.0 & 0.0 & 2.7 & 0.0 & 0.0 & 0.0 & 0.0 & 0.0 \\
\hline 22 & $207-216$ & $\begin{array}{l}207.06- \\
215.21\end{array}$ & 89.3 & 83.3 & 14.9 & 9.9 & 14.2 & 25.3 & 34.2 & 0.0 & 0.0 & 0.0 & 1.6 & 0.0 & 0.0 & 0.0 & 0.0 & 0.0 \\
\hline
\end{tabular}


TABLE 2 - Continued

\begin{tabular}{|c|c|c|c|c|c|c|c|c|c|c|c|c|c|c|c|c|c|c|}
\hline \multicolumn{19}{|c|}{ Hole 62.1 - Continued } \\
\hline Core & $\begin{array}{l}\text { Downhole } \\
\text { Depth of } \\
\text { Core }\end{array}$ & $\begin{array}{c}\text { Depth } \\
\text { Range } \\
\text { Sampled }\end{array}$ & Diff. & $\begin{array}{c}\% \\
\text { Amorphous }\end{array}$ & Quar. & Plag. & Kaol. & Mica & Mont. & Crist. & Clin. & $\mathrm{K}-\mathrm{Fe}$ & Pyrite & Barite & Apat. & Side. & Paly. & Phil. \\
\hline 23 & $216-225$ & $\begin{array}{l}216.10- \\
224.21\end{array}$ & 87.9 & 81.1 & 7.8 & 6.8 & 7.5 & 13.1 & 64.8 & 0.0 & 0.0 & 0.0 & 0.0 & 0.0 & 0.0 & 0.0 & 0.0 & 0.0 \\
\hline 24 & $225-234$ & $\begin{array}{l}225.07- \\
233.21\end{array}$ & 88.2 & 81.6 & 8.5 & 3.8 & 9.3 & 13.9 & 61.7 & 0.0 & 0.0 & 0.0 & 2.2 & 0.0 & 0.0 & 0.0 & 0.0 & 0.0 \\
\hline 25 & $234-243$ & $\begin{array}{l}234.09- \\
242.21\end{array}$ & 88.9 & 82.7 & 9.0 & 4.2 & 8.6 & 13.2 & 63.7 & 0.0 & 0.0 & 0.0 & 1.5 & 0.0 & 0.0 & 0.0 & 0.0 & 0.0 \\
\hline 26 & $245-254$ & $\begin{array}{l}245.10- \\
253.21\end{array}$ & 88.6 & 82.2 & 6.9 & 3.6 & 8.5 & 14.6 & 66.4 & 0.0 & 0.0 & 0.0 & 0.0 & 0.0 & 0.0 & 0.0 & 0.0 & 0.0 \\
\hline 27 & $254-263$ & $\begin{array}{l}255.11- \\
260.71\end{array}$ & 90.2 & 84.7 & 13.4 & 10.0 & 14.3 & 17.7 & 44.6 & 0.0 & 0.0 & 0.0 & 0.0 & 0.0 & 0.0 & 0.0 & 0.0 & 0.0 \\
\hline 28 & $263-271$ & $\begin{array}{l}263.06- \\
271.00\end{array}$ & 90.4 & 85.0 & 8.4 & 8.6 & 11.5 & 21.1 & 50.4 & 0.0 & 0.0 & 0.0 & 0.0 & 0.0 & 0.0 & 0.0 & 0.0 & 0.0 \\
\hline 29 & $271-281$ & $\begin{array}{l}271.06- \\
279.21\end{array}$ & 90.0 & 84.4 & 7.0 & 6.8 & 7.8 & 0.0 & 78.4 & 0.0 & 0.0 & 0.0 & 0.0 & 0.0 & 0.0 & 0.0 & 0.0 & 0.0 \\
\hline 30 & $281-291$ & $\begin{array}{l}281.05- \\
289.21\end{array}$ & 87.1 & 79.8 & 3.8 & 2.2 & 4.4 & 0.0 & 89.3 & 0.0 & 0.0 & 0.0 & 0.0 & 0.0 & 0.0 & 0.0 & 0.0 & 0.0 \\
\hline 31 & $291-300$ & $\begin{array}{l}291.02- \\
299.21\end{array}$ & 88.6 & 82.2 & 3.6 & 4.3 & 6.8 & 11.9 & 72.4 & 0.0 & 0.0 & 0.0 & 0.0 & 0.0 & 0.0 & 0.0 & 0.0 & 0.0 \\
\hline 32 & $301-310$ & $\begin{array}{l}301.04- \\
309.21\end{array}$ & 86.7 & 79.2 & 6.8 & 6.0 & 11.3 & 14.2 & 61.6 & 0.0 & 0.0 & 0.0 & 0.0 & 0.0 & 0.0 & 0.0 & 0.0 & 0.0 \\
\hline 33 & $310-319$ & $\begin{array}{l}311.55- \\
313.71\end{array}$ & 86.2 & 78.4 & 5.4 & 3.3 & 6.7 & 11.2 & 73.2 & 0.0 & 0.0 & 0.0 & 0.0 & 0.0 & 0.0 & 0.0 & 0.0 & 0.0 \\
\hline 34 & $320-327 *$ & $\begin{array}{l}319.07- \\
327.00 *\end{array}$ & 87.6 & 80.6 & 7.3 & 2.3 & 7.6 & 9.5 & 73.4 & 0.0 & 0.0 & 0.0 & 0.0 & 0.0 & 0.0 & 0.0 & 0.0 & 0.0 \\
\hline 35 & $327-336$ & $\begin{array}{l}327.33- \\
335.21\end{array}$ & 87.6 & 80.6 & 5.4 & 1.9 & 6.1 & 11.7 & 74.9 & 0.0 & 0.0 & 0.0 & 0.0 & 0.0 & 0.0 & 0.0 & 0.0 & 0.0 \\
\hline 36 & $336-344$ & $\begin{array}{l}337.55- \\
342.71\end{array}$ & 88.7 & 82.3 & 11.9 & 6.6 & 13.9 & 15.0 & 52.7 & 0.0 & 0.0 & 0.0 & 0.0 & 0.0 & 0.0 & 0.0 & 0.0 & 0.0 \\
\hline 37 & $344-350$ & $\begin{array}{l}345.55- \\
346.21\end{array}$ & 86.6 & 79.1 & 8.5 & 6.5 & 7.1 & 23.8 & 54.2 & 0.0 & 0.0 & 0.0 & 0.0 & 0.0 & 0.0 & 0.0 & 0.0 & 0.0 \\
\hline
\end{tabular}




\begin{tabular}{|c|c|c|c|c|c|c|c|c|c|c|c|c|c|c|c|c|c|c|}
\hline \multicolumn{19}{|c|}{ Hole 63.0} \\
\hline Core & $\begin{array}{l}\text { Downhole } \\
\text { Depth of } \\
\text { Core }\end{array}$ & $\begin{array}{c}\text { Depth } \\
\text { Range } \\
\text { Sampled }\end{array}$ & Diff. & $\begin{array}{c}\% \\
\text { Amorphous }\end{array}$ & Quar. & Plag. & Kaol. & Mica & Mont. & Crist. & Clin. & $\mathrm{K}-\mathrm{Fe}$ & Pyrite & Barite & Apat. & Side. & Paly. & Phil. \\
\hline 1 & $0-9$ & $\begin{array}{l}0.06- \\
8.22\end{array}$ & 83.9 & 74.8 & 6.5 & 6.8 & 13.6 & 43.7 & 29.4 & 0.0 & 0.0 & 0.0 & 0.0 & 0.0 & 0.0 & 0.0 & 0.0 & 0.0 \\
\hline 2 & $61-70$ & $\begin{array}{l}62.55- \\
69.21\end{array}$ & 90.4 & 85.0 & 6.9 & 6.1 & 10.3 & 14.4 & 62.3 & 0.0 & 0.0 & 0.0 & 0.0 & 0.0 & 0.0 & 0.0 & 0.0 & 0.0 \\
\hline 3 & $137-146$ & $\begin{array}{l}137.50- \\
142.21\end{array}$ & 88.4 & 81.9 & 9.8 & 6.2 & 12.9 & 27.1 & 44.1 & 0.0 & 0.0 & 0.0 & 0.0 & 0.0 & 0.0 & 0.0 & 0.0 & 0.0 \\
\hline 4 & $230-240$ & $\begin{array}{l}230.10- \\
232.21\end{array}$ & 84.5 & 75.8 & 2.3 & 2.6 & 3.0 & 8.9 & 83.1 & 0.0 & 0.0 & 0.0 & 0.0 & 0.0 & 0.0 & 0.0 & 0.0 & 0.0 \\
\hline 5 & $352-359$ & $\begin{array}{l}352.14- \\
354.22\end{array}$ & 84.0 & 75.0 & 1.8 & 6.0 & 0.0 & 0.0 & 92.2 & 0.0 & 0.0 & 0.0 & 0.0 & 0.0 & 0.0 & 0.0 & 0.0 & 0.0 \\
\hline 6 & $458-467$ & $\begin{array}{l}458.06- \\
466.21\end{array}$ & 86.5 & 78.9 & 11.3 & 3.2 & 0.0 & 0.0 & 71.3 & 0.0 & 14.3 & 0.0 & 0.0 & 0.0 & 0.0 & 0.0 & 0.0 & 0.0 \\
\hline 7 & $534-543$ & $\begin{array}{l}534.04 \\
542.21\end{array}$ & 88.6 & 66.5 & 42.1 & 0.0 & 0.0 & 16.1 & 41.8 & 0.0 & 0.0 & 0.0 & 0.0 & 0.0 & 0.0 & 0.0 & 0.0 & 0.0 \\
\hline 8 & $543-553$ & $\begin{array}{l}543.52- \\
547.49\end{array}$ & 85.8 & 77.8 & 15.3 & 11.9 & 15.9 & 30.5 & 26.4 & 0.0 & 0.0 & 0.0 & 0.0 & 0.0 & 0.0 & 0.0 & 0.0 & 0.0 \\
\hline 9 & $553-561$ & $\begin{array}{l}553.43- \\
557.64\end{array}$ & 93.6 & 81.2 & 10.2 & 0.0 & 0.0 & 0.0 & 80.4 & 9.4 & 0.0 & 0.0 & 0.0 & 0.0 & 0.0 & 0.0 & 0.0 & 0.0 \\
\hline \multicolumn{19}{|c|}{ Hole 63.1} \\
\hline Core & $\begin{array}{l}\text { Downhole } \\
\text { Depth of } \\
\text { Core }\end{array}$ & $\begin{array}{c}\text { Depth } \\
\text { Range } \\
\text { Sampled }\end{array}$ & Diff. & $\begin{array}{c}\% \\
\text { Amorphous }\end{array}$ & Quar. & Plag. & Kaol. & Mica & Mont. & Crist. & Clin. & $\mathrm{K}-\mathrm{Fe}$ & Pyrite & Barite & Apat. & Side. & Paly. & Phil. \\
\hline 1 & $3-13$ & $\begin{array}{l}3.50 \\
8.10\end{array}$ & 83.3 & 73.9 & 17.2 & 6.4 & 11.1 & 42.0 & 23.2 & 0.0 & 0.0 & 0.0 & 0.0 & 0.0 & 0.0 & 0.0 & 0.0 & 0.0 \\
\hline 3 & $22-32$ & $\begin{array}{l}22.58- \\
24.21\end{array}$ & 82.4 & 72.5 & 7.4 & 7.9 & 10.8 & 39.0 & 34.8 & 0.0 & 0.0 & 0.0 & 0.0 & 0.0 & 0.0 & 0.0 & 0.0 & 0.0 \\
\hline 5 & $101-110$ & $\begin{array}{l}101.55- \\
106.21\end{array}$ & 89.4 & 83.4 & 5.6 & 7.0 & 11.1 & 21.2 & 55.2 & 0.0 & 0.0 & 0.0 & 0.0 & 0.0 & 0.0 & 0.0 & 0.0 & 0.0 \\
\hline
\end{tabular}


TABLE 2 - Continued

\begin{tabular}{|c|c|c|c|c|c|c|c|c|c|c|c|c|c|c|c|c|c|c|}
\hline \multicolumn{19}{|c|}{ Hole 63.1 - Continued } \\
\hline Core & $\begin{array}{c}\text { Downhole } \\
\text { Depth of } \\
\text { Core }\end{array}$ & $\begin{array}{l}\text { Depth } \\
\text { Range } \\
\text { Sampled }\end{array}$ & Diff. & $\begin{array}{c}\% \\
\text { Amorphous }\end{array}$ & Quar. & Plag. & Kaol. & Mica & Mont. & Crist. & Clin. & $\mathrm{K}-\mathrm{Fe}$ & Pyrite & Barite & Apat. & Side. & Paly. & Phil. \\
\hline 6 & $110-119$ & $\begin{array}{l}110.65- \\
118.22\end{array}$ & 88.1 & 81.4 & 6.6 & 7.6 & 12.1 & 13.8 & 59.9 & 0.0 & 0.0 & 0.0 & 0.0 & 0.0 & 0.0 & 0.0 & 0.0 & 0.0 \\
\hline 7 & $119-129$ & $\begin{array}{l}119.05- \\
122.71\end{array}$ & 89.0 & 82.8 & 6.5 & 8.2 & 12.9 & 13.6 & 58.8 & 0.0 & 0.0 & 0.0 & 0.0 & 0.0 & 0.0 & 0.0 & 0.0 & 0.0 \\
\hline 8 & $129-138$ & $\begin{array}{l}130.63- \\
137.21\end{array}$ & 89.5 & 83.6 & 8.5 & 8.6 & 14.0 & 11.7 & 57.3 & 0.0 & 0.0 & 0.0 & 0.0 & 0.0 & 0.0 & 0.0 & 0.0 & 0.0 \\
\hline 9 & $138-148$ & $\begin{array}{l}138.12- \\
146.21\end{array}$ & 89.9 & 84.2 & 7.6 & 4.8 & 15.1 & 21.1 & 51.4 & 0.0 & 0.0 & 0.0 & 0.0 & 0.0 & 0.0 & 0.0 & 0.0 & 0.0 \\
\hline 10 & $148-155$ & $\begin{array}{l}148.16- \\
154.76\end{array}$ & 88.7 & 82.3 & 8.7 & 4.4 & 11.5 & 24.2 & 51.2 & 0.0 & 0.0 & 0.0 & 0.0 & 0.0 & 0.0 & 0.0 & 0.0 & 0.0 \\
\hline 11 & $155-165$ & $\begin{array}{l}155.03- \\
163.21\end{array}$ & 88.7 & 82.3 & 9.9 & 7.5 & 17.8 & 20.4 & 44.3 & 0.0 & 0.0 & 0.0 & 0.0 & 0.0 & 0.0 & 0.0 & 0.0 & 0.0 \\
\hline 12 & $165-174$ & $\begin{array}{l}166.62- \\
167.21\end{array}$ & 88.1 & 81.4 & 8.2 & 3.3 & 12.3 & 24.8 & 51.3 & 0.0 & 0.0 & 0.0 & 0.0 & 0.0 & 0.0 & 0.0 & 0.0 & 0.0 \\
\hline 13 & $174-184$ & $\begin{array}{l}174.05- \\
182.00\end{array}$ & 87.4 & 80.3 & 10.0 & 4.9 & 12.8 & 10.8 & 61.5 & 0.0 & 0.0 & 0.0 & 0.0 & 0.0 & 0.0 & 0.0 & 0.0 & 0.0 \\
\hline 14 & $184-193$ & $\begin{array}{l}184.06- \\
192.33\end{array}$ & 86.3 & 78.6 & 6.6 & 5.3 & 8.3 & 6.7 & 73.1 & 0.0 & 0.0 & 0.0 & 0.0 & 0.0 & 0.0 & 0.0 & 0.0 & 0.0 \\
\hline \multicolumn{19}{|c|}{ Hole 63.2} \\
\hline Core & $\begin{array}{l}\text { Downhole } \\
\text { Depth of } \\
\text { Core }\end{array}$ & $\begin{array}{l}\text { Depth } \\
\text { Range } \\
\text { Sampled }\end{array}$ & Diff. & $\begin{array}{c}\% \\
\text { Amorphous }\end{array}$ & Quar. & Plag. & Kaol. & Mica & Mont. & Crist. & Clin. & $\mathrm{K}-\mathrm{Fe}$ & Pyrite & Barite & Apat. & Side. & Paly. & Phil. \\
\hline 1 & $11-20$ & $\begin{array}{l}11.05- \\
16.21\end{array}$ & 85.3 & 77.0 & 5.4 & 5.1 & 10.8 & 38.0 & 40.8 & 0.0 & 0.0 & 0.0 & 0.0 & 0.0 & 0.0 & 0.0 & 0.0 & 0.0 \\
\hline 2 & $20-30$ & $\begin{array}{l}20.05- \\
28.67\end{array}$ & 88.6 & 82.2 & 13.0 & 11.7 & 6.3 & 56.6 & 7.4 & 0.0 & 0.0 & 0.0 & 0.0 & 0.0 & 0.0 & 0.0 & 0.0 & 0.0 \\
\hline 3 & $30-39$ & $\begin{array}{l}30.42- \\
35.21\end{array}$ & 85.7 & 77.7 & 16.8 & 17.1 & 17.0 & 24.5 & 24.7 & 0.0 & 0.0 & 0.0 & 0.0 & 0.0 & 0.0 & 0.0 & 0.0 & 0.0 \\
\hline
\end{tabular}




\begin{tabular}{|c|c|c|c|c|c|c|c|c|c|c|c|c|c|c|c|c|c|c|}
\hline \multicolumn{19}{|c|}{ Hole 64.0} \\
\hline Core & $\begin{array}{l}\text { Downhole } \\
\text { Depth of } \\
\text { Core }\end{array}$ & $\begin{array}{c}\text { Depth } \\
\text { Range } \\
\text { Sampled }\end{array}$ & Diff. & $\begin{array}{c}\% \\
\text { Amorphous }\end{array}$ & Quar. & Plag. & Kaol. & Mica & Mont. & Crist. & Clin. & $\mathrm{K}-\mathrm{Fe}$ & Pyrite & Barite & Apat. & Side. & Paly. & Phil \\
\hline 1 & $0-9$ & $\begin{array}{l}0.04- \\
8.51\end{array}$ & 87.4 & 80.3 & 3.5 & 6.9 & 7.8 & 16.4 & 65.3 & 0.0 & 0.0 & 0.0 & 0.0 & 0.0 & 0.0 & 0.0 & 0.0 & 0.0 \\
\hline 2 & $99-108$ & $\begin{array}{c}99.37- \\
107.21\end{array}$ & 89.5 & 83.6 & 2.6 & 5.4 & 9.5 & 23.7 & 58.8 & 0.0 & 0.0 & 0.0 & 0.0 & 0.0 & 0.0 & 0.0 & 0.0 & 0.0 \\
\hline 3 & $202-211$ & $\begin{array}{l}202.04- \\
210.21\end{array}$ & 89.9 & 84.2 & 3.8 & 7.4 & 14.5 & 20.3 & 44.1 & 8.7 & 0.0 & 0.0 & 0.0 & 0.0 & 0.0 & 0.0 & 0.0 & 0.0 \\
\hline 4 & $304-313$ & $\begin{array}{l}304.51- \\
312.21\end{array}$ & 91.6 & 86.9 & 3.8 & 11.6 & 17.0 & 0.0 & 51.5 & 11.8 & 0.0 & 0.0 & 0.0 & 4.4 & 0.0 & 0.0 & 0.0 & 0.0 \\
\hline 5 & $409-418$ & $\begin{array}{l}409.11- \\
417.29\end{array}$ & 87.7 & 80.8 & 4.3 & 6.1 & 12.6 & 14.4 & 62.5 & 0.0 & 0.0 & 0.0 & 0.0 & 0.0 & 0.0 & 0.0 & 0.0 & 0.0 \\
\hline 6 & $505-514$ & $\begin{array}{l}505.08- \\
513.22\end{array}$ & 88.8 & 82.5 & 1.6 & 2.8 & 6.8 & 15.3 & 66.3 & 0.0 & 0.0 & 0.0 & 7.3 & 0.0 & 0.0 & 0.0 & 0.0 & 0.0 \\
\hline 7 & $610-619$ & $\begin{array}{l}610.25- \\
618.22\end{array}$ & 89.7 & 83.9 & 1.6 & 4.5 & 5.6 & 11.8 & 76.5 & 0.0 & 0.0 & 0.0 & 0.0 & 0.0 & 0.0 & 0.0 & 0.0 & 0.0 \\
\hline 8 & $705-710$ & $\begin{array}{l}\text { 705.37- } \\
708.71\end{array}$ & 93.2 & 89.4 & 0.0 & 21.9 & 0.0 & 0.0 & 78.1 & 0.0 & 0.0 & 0.0 & 0.0 & 0.0 & 0.0 & 0.0 & 0.0 & 0.0 \\
\hline 10 & $848-851$ & $\begin{array}{l}848.82- \\
850.21\end{array}$ & 89.6 & 83.8 & 2.2 & 11.6 & 3.3 & 0.0 & 82.8 & 0.0 & 0.0 & 0.0 & 0.0 & 0.0 & 0.0 & 0.0 & 0.0 & 0.0 \\
\hline \multicolumn{19}{|c|}{ Hole 64.1} \\
\hline Core & $\begin{array}{l}\text { Downhole } \\
\text { Depth of } \\
\text { Core }\end{array}$ & $\begin{array}{c}\text { Depth } \\
\text { Range } \\
\text { Sampled }\end{array}$ & Diff. & $\begin{array}{c}\% \\
\text { Amorphous }\end{array}$ & Quar. & Plag. & Kaol. & Mica & Mont. & Crist. & Clin. & $\mathrm{K}-\mathrm{Fe}$ & Pyrite & Barite & Apat. & Side. & Paly. & Phil. \\
\hline 1 & $433-442$ & $\begin{array}{l}433.13- \\
441.22\end{array}$ & 90.8 & 85.6 & 4.3 & 9.6 & 12.3 & 14.4 & 50.7 & 0.0 & 0.0 & 0.0 & 0.0 & 8.8 & 0.0 & 0.0 & 0.0 & 0.0 \\
\hline 2 & $442-451$ & $\begin{array}{l}442.30- \\
450.21\end{array}$ & 91.1 & 86.1 & 4.8 & 11.7 & 11.3 & 19.8 & 39.7 & 0.0 & 0.0 & 0.0 & 0.0 & 12.6 & 0.0 & 0.0 & 0.0 & 0.0 \\
\hline 3 & $451-461$ & $\begin{array}{l}451.13- \\
459.19\end{array}$ & 90.0 & 84.4 & 2.1 & 5.7 & 7.8 & 40.7 & 36.6 & 0.0 & 0.0 & 0.0 & 0.0 & 7.1 & 0.0 & 0.0 & 0.0 & 0.0 \\
\hline
\end{tabular}


TABLE 2 - Continued

\begin{tabular}{|c|c|c|c|c|c|c|c|c|c|c|c|c|c|c|c|c|c|c|}
\hline \multicolumn{19}{|c|}{ Hole 64.1 - Continued } \\
\hline Core & $\begin{array}{l}\text { Downhole } \\
\text { Depth of } \\
\text { Core }\end{array}$ & $\begin{array}{l}\text { Depth } \\
\text { Range } \\
\text { Sampled }\end{array}$ & Diff. & $\begin{array}{c}\% \\
\text { Amorphous }\end{array}$ & Quar. & Plag. & Kaol. & Mica & Mont. & Crist. & Clin. & $\mathrm{K} \cdot \mathrm{Fe}$ & Pyrite & Barite & Apat. & Side. & Paly. & Phil. \\
\hline 4 & $461-470$ & $\begin{array}{l}461.13- \\
469.21\end{array}$ & 90.4 & 85.0 & 3.9 & 7.1 & 11.1 & 14.5 & 56.8 & 0.0 & 0.0 & 0.0 & 0.0 & 6.6 & 0.0 & 0.0 & 0.0 & 0.0 \\
\hline 5 & $470-479$ & $\begin{array}{l}470.29- \\
478.21\end{array}$ & 90.1 & 84.5 & 3.7 & 6.5 & 9.5 & 17.7 & 57.7 & 0.0 & 0.0 & 0.0 & 0.0 & 4.9 & 0.0 & 0.0 & 0.0 & 0.0 \\
\hline 6 & $565-571$ & $\begin{array}{l}565.06- \\
570.31\end{array}$ & 90.1 & 84.5 & 2.1 & 7.9 & 9.7 & 0.0 & 76.0 & 0.0 & 0.0 & 0.0 & 0.0 & 4.3 & 0.0 & 0.0 & 0.0 & 0.0 \\
\hline 7 & $661-663^{*}$ & $\begin{array}{l}662.03- \\
666.22 *\end{array}$ & 91.0 & 85.9 & 2.3 & 12.8 & 5.8 & 0.0 & 79.0 & 0.0 & 0.0 & 0.0 & 0.0 & 0.0 & 0.0 & 0.0 & 0.0 & 0.0 \\
\hline 9 & $911-916$ & $\begin{array}{l}911.12- \\
914.72\end{array}$ & 92.8 & 88.8 & 5.2 & 12.0 & 0.0 & 0.0 & 46.7 & 0.0 & 0.0 & 0.0 & 3.8 & 5.7 & 26.6 & 0.0 & 0.0 & 0.0 \\
\hline 10 & $969-972$ & $\begin{array}{l}969.16- \\
971.21\end{array}$ & 92.2 & 87.8 & 5.7 & 8.8 & 0.0 & 0.0 & 47.1 & 0.0 & 0.0 & 0.0 & 0.0 & 11.5 & 26.8 & 0.0 & 0.0 & 0.0 \\
\hline \multicolumn{19}{|c|}{ Hole 65.0} \\
\hline Core & $\begin{array}{l}\text { Downhole } \\
\text { Depth of } \\
\text { Core }\end{array}$ & $\begin{array}{l}\text { Depth } \\
\text { Range } \\
\text { Sampled }\end{array}$ & Diff. & $\begin{array}{c}\% \\
\text { Amorphous }\end{array}$ & Quar. & Plag. & Kaol. & Mica & Mont. & Crist. & Clin. & $\mathrm{K}-\mathrm{Fe}$ & Pyrite & Barite & Apat. & Side. & Paly. & Phil. \\
\hline 2 & $10-19$ & $\begin{array}{l}10.42= \\
16.91\end{array}$ & 93.3 & 89.5 & 7.3 & 12.7 & 7.7 & 26.8 & 45.4 & 0.0 & 0.0 & 0.0 & 0.0 & 0.0 & 0.0 & 0.0 & 0.0 & 0.0 \\
\hline 3 & $19-28$ & $\begin{array}{l}19.19- \\
25.71\end{array}$ & 92.3 & 88.0 & 11.6 & 11.1 & 9.8 & 41.0 & 26.6 & 0.0 & 0.0 & 0.0 & 0.0 & 0.0 & 0.0 & 0.0 & 0.0 & 0.0 \\
\hline 4 & $28-37$ & $\begin{array}{l}29.65- \\
34.71\end{array}$ & 92.1 & 87.7 & 8.7 & 12.0 & 7.2 & 20.7 & 51.4 & 0.0 & 0.0 & 0.0 & 0.0 & 0.0 & 0.0 & 0.0 & 0.0 & 0.0 \\
\hline 5 & $37-46$ & $\begin{array}{l}38.92- \\
45.21\end{array}$ & 92.4 & 88.1 & 13.6 & 15.8 & 0.0 & 0.0 & 70.5 & 0.0 & 0.0 & 0.0 & 0.0 & 0.0 & 0.0 & 0.0 & 0.0 & 0.0 \\
\hline 6 & $46-55$ & $\begin{array}{l}47.94- \\
48.92\end{array}$ & 94.0 & 90.6 & 6.8 & 26.2 & 8.5 & 21.5 & 37.0 & 0.0 & 0.0 & 0.0 & 0.0 & 0.0 & 0.0 & 0.0 & 0.0 & 0.0 \\
\hline 7 & $55-64$ & $\begin{array}{l}56.89- \\
61.92\end{array}$ & 91.3 & 86.4 & 9.4 & 10.2 & 7.4 & 0.0 & 73.0 & 0.0 & 0.0 & 0.0 & 0.0 & 0.0 & 0.0 & 0.0 & 0.0 & 0.0 \\
\hline
\end{tabular}


TABLE 2 - Continued

\begin{tabular}{|c|c|c|c|c|c|c|c|c|c|c|c|c|c|c|c|c|c|c|}
\hline \multicolumn{19}{|c|}{ Hole 65.0 - Continued } \\
\hline Core & $\begin{array}{l}\text { Downhole } \\
\text { Depth of } \\
\text { Core }\end{array}$ & $\begin{array}{c}\text { Depth } \\
\text { Range } \\
\text { Sampled }\end{array}$ & Diff. & $\begin{array}{c}\% \\
\text { Amorphous }\end{array}$ & Quar. & Plag. & Kaol. & Mica & Mont. & Crist. & Clin. & $\mathrm{K}-\mathrm{Fe}$ & Pyrite & Barite & Apat. & Side. & Paly & Phil. \\
\hline 8 & $64-74$ & $\begin{array}{l}64.10- \\
72.21\end{array}$ & 90.5 & 85.2 & 12.4 & 10.4 & 10.7 & 38.2 & 28.2 & 0.0 & 0.0 & 0.0 & 0.0 & 0.0 & 0.0 & 0.0 & 0.0 & 0.0 \\
\hline 9 & $74-83$ & $\begin{array}{l}74.24- \\
82.41\end{array}$ & 90.7 & 85.5 & 8.0 & 9.9 & 12.1 & 22.5 & 47.6 & 0.0 & 0.0 & 0.0 & 0.0 & 0.0 & 0.0 & 0.0 & 0.0 & 0.0 \\
\hline 10 & $83-92$ & $\begin{array}{l}83.73- \\
88.21\end{array}$ & 92.0 & 87.5 & 29.0 & 40.4 & 30.5 & 0.0 & 0.0 & 0.0 & 0.0 & 0.0 & 0.0 & 0.0 & 0.0 & 0.0 & 0.0 & 0.0 \\
\hline 11 & $92-101$ & $\begin{array}{c}94.18- \\
100.52\end{array}$ & 93.5 & 89.8 & 28.1 & 71.9 & 0.0 & 0.0 & 0.0 & 0.0 & 0.0 & 0.0 & 0.0 & 0.0 & 0.0 & 0.0 & 0.0 & 0.0 \\
\hline 12 & $101-110$ & $\begin{array}{l}101.07- \\
109.40\end{array}$ & 88.8 & 82.5 & 5.7 & 19.2 & 0.0 & 0.0 & 44.0 & 0.0 & 31.0 & 0.0 & 0.0 & 0.0 & 0.0 & 0.0 & 0.0 & 0.0 \\
\hline 13 & $110-119$ & $\begin{array}{l}110.34- \\
118.41\end{array}$ & 92.3 & 88.0 & 15.8 & 60.5 & 23.7 & 0.0 & 0.0 & 0.0 & 0.0 & 0.0 & 0.0 & 0.0 & 0.0 & 0.0 & 0.0 & 0.0 \\
\hline 14 & $119-127 *$ & $\begin{array}{l}119.06- \\
127.41^{*}\end{array}$ & 95.6 & 87.1 & 5.2 & 8.1 & 0.0 & 0.0 & 76.4 & 0.0 & 4.1 & 0.0 & 0.0 & 0.0 & 0.0 & 6.1 & 0.0 & 0.0 \\
\hline 16 & $137-145^{*}$ & $\begin{array}{l}\text { 138.97- } \\
145.21^{*}\end{array}$ & 89.7 & 83.9 & 40.5 & 21.9 & 0.0 & 0.0 & 0.0 & 0.0 & 37.6 & 0.0 & 0.0 & 0.0 & 0.0 & 0.0 & 0.0 & 0.0 \\
\hline \multicolumn{19}{|c|}{ Hole 65.1} \\
\hline Core & $\begin{array}{l}\text { Downhole } \\
\text { Depth of } \\
\text { Core }\end{array}$ & $\begin{array}{c}\text { Depth } \\
\text { Range } \\
\text { Sampled }\end{array}$ & Diff. & $\begin{array}{c}\% \\
\text { Amorphous }\end{array}$ & Quar. & Plag. & Kaol. & Mica & Mont. & Crist. & Clin. & $\mathrm{K} \cdot \mathrm{Fe}$ & Pyrite & Barite & Apat. & Side. & Paly. & Phil. \\
\hline 2 & $145-147$ & $\begin{array}{l}146.01- \\
146.35\end{array}$ & 89.4 & 83.4 & 18.2 & 4.4 & 3.4 & 28.9 & 28.7 & 0.0 & 16.4 & 0.0 & 0.0 & 0.0 & 0.0 & 0.0 & 0.0 & 0.0 \\
\hline 4 & $154-162 *$ & $\begin{array}{l}153.37- \\
161.21\end{array}$ & 90.6 & 85.6 & 35.6 & 13.6 & 5.6 & 32.7 & 12.5 & 0.0 & 0.0 & 0.0 & 0.0 & 0.0 & 0.0 & 0.0 & 0.0 & 0.0 \\
\hline 5 & $162-168$ & $\begin{array}{l}162.61- \\
167.88\end{array}$ & 91.4 & 86.6 & 4.4 & 4.6 & 0.0 & 0.0 & 90.9 & 0.0 & 0.0 & 0.0 & 0.0 & 0.0 & 0.0 & 0.0 & 0.0 & 0.0 \\
\hline
\end{tabular}


TABLE 2 - Continued

\begin{tabular}{|c|c|c|c|c|c|c|c|c|c|c|c|c|c|c|c|c|c|c|}
\hline \multicolumn{19}{|c|}{ Hole 66.0} \\
\hline Core & $\begin{array}{l}\text { Downhole } \\
\text { Depth of } \\
\text { Core }\end{array}$ & $\begin{array}{c}\text { Depth } \\
\text { Range } \\
\text { Sampled }\end{array}$ & Diff. & $\begin{array}{c}\% \\
\text { Amorphous }\end{array}$ & Quar. & Plag. & Kaol. & Mica & Mont. & Crist. & Clin. & $\mathrm{K}-\mathrm{Fe}$ & Pyrite & Barite & Apat. & Side. & Paly. & Phil. \\
\hline 3 & $117-126$ & $\begin{array}{l}117.54- \\
125.41\end{array}$ & 92.5 & 88.3 & 17.2 & 26.9 & 11.7 & 44.2 & 0.0 & 0.0 & 0.0 & 0.0 & 0.0 & 0.0 & 0.0 & 0.0 & 0.0 & 0.0 \\
\hline 6 & $165-174$ & $\begin{array}{l}165.09 . \\
170.21\end{array}$ & 77.9 & 65.5 & 4.5 & 0.0 & 1.2 & 53.2 & 4.8 & 0.0 & 0.0 & 6.2 & 0.0 & 0.0 & 0.0 & 0.0 & 30.1 & 0.0 \\
\hline 7 & $174-180$ & $\begin{array}{l}174.33- \\
179.21\end{array}$ & 77.2 & 64.4 & 6.1 & 0.0 & 3.5 & 49.3 & 8.1 & 0.0 & 0.0 & 6.1 & 0.0 & 0.0 & 0.0 & 0.0 & 26.8 & 0.0 \\
\hline 8 & $180-187$ & $\begin{array}{l}180.12- \\
186.29\end{array}$ & 82.0 & 71.9 & 5.7 & 0.0 & 3.2 & 47.7 & 2.5 & 0.0 & 0.0 & 6.4 & 0.0 & 0.0 & 0.0 & 0.0 & 34.5 & 0.0 \\
\hline \multicolumn{19}{|c|}{ Hole 66.1} \\
\hline Core & $\begin{array}{l}\text { Downhole } \\
\text { Depth of } \\
\text { Core }\end{array}$ & $\begin{array}{c}\text { Depth } \\
\text { Range } \\
\text { Sampled }\end{array}$ & Diff. & $\begin{array}{c}\% \\
\text { Amorphous }\end{array}$ & Quar. & Plag. & Kaol. & Mica & Mont. & Crist. & Clin. & $\mathrm{K}-\mathrm{Fe}$ & Pyrite & Barite & Apat. & Side. & Paly. & Phi. \\
\hline 2 & $20-29$ & $\begin{array}{l}20.11- \\
28.21\end{array}$ & 94.4 & 91.2 & 6.0 & 16.2 & 6.3 & 39.9 & 31.6 & 0.0 & 0.0 & 0.0 & 0.0 & 0.0 & 0.0 & 0.0 & 0.0 & 0.0 \\
\hline 3 & $29-38$ & $\begin{array}{l}29.26- \\
37.19\end{array}$ & 94.0 & 90.6 & 7.0 & 16.2 & 6.3 & 35.4 & 35.1 & 0.0 & 0.0 & 0.0 & 0.0 & 0.0 & 0.0 & 0.0 & 0.0 & 0.0 \\
\hline 4 & $38-47$ & $\begin{array}{l}39.04- \\
46.42\end{array}$ & 95.0 & 92.2 & 6.8 & 15.7 & 0.0 & 43.2 & 34.3 & 0.0 & 0.0 & 0.0 & 0.0 & 0.0 & 0.0 & 0.0 & 0.0 & 0.0 \\
\hline 5 & $47-56$ & $\begin{array}{l}47.06- \\
55.31\end{array}$ & 95.7 & 93.3 & 9.0 & 20.0 & 0.0 & 0.0 & 71.0 & 0.0 & 0.0 & 0.0 & 0.0 & 0.0 & 0.0 & 0.0 & 0.0 & 0.0 \\
\hline 6 & $56-65$ & $\begin{array}{l}57.65- \\
64.32\end{array}$ & 94.8 & 91.9 & 100.0 & 0.0 & 0.0 & 0.0 & 0.0 & 0.0 & 0.0 & 0.0 & 0.0 & 0.0 & 0.0 & 0.0 & 0.0 & 0.0 \\
\hline 7 & $67-76$ & $\begin{array}{l}67.14- \\
75.32\end{array}$ & 95.3 & 92.9 & 100.0 & 0.0 & 0.0 & 0.0 & 0.0 & 0.0 & 0.0 & 0.0 & 0.0 & 0.0 & 0.0 & 0.0 & 0.0 & 0.0 \\
\hline 8 & $76-86$ & $\begin{array}{l}76.05- \\
84.31\end{array}$ & 95.1 & 92.3 & 21.0 & 48.0 & 31.0 & 0.0 & 0.0 & 0.0 & 0.0 & 0.0 & 0.0 & 0.0 & 0.0 & 0.0 & 0.0 & 0.0 \\
\hline
\end{tabular}


TABLE 2 - Continued

\begin{tabular}{|c|c|c|c|c|c|c|c|c|c|c|c|c|c|c|c|c|c|c|}
\hline \multicolumn{19}{|c|}{ Hole 67.0} \\
\hline Core & $\begin{array}{l}\text { Downhole } \\
\text { Depth of } \\
\text { Core }\end{array}$ & $\begin{array}{c}\text { Depth } \\
\text { Range } \\
\text { Sampled }\end{array}$ & Diff. & $\begin{array}{c}\% \\
\text { Amorphous }\end{array}$ & Quar. & Plag. & Kaol. & Mica & Mont. & Crist. & Clin. & $\mathrm{K}-\mathrm{Fe}$ & Pyrite & Barite & Apat. & Side. & Paly. & Phil. \\
\hline 1 & $0-5$ & $\begin{array}{l}0.31- \\
1.09\end{array}$ & 88.4 & 81.9 & 8.7 & 14.5 & 3.3 & 34.5 & 0.0 & 0.0 & 0.0 & 0.0 & 0.0 & 0.0 & 0.0 & 0.0 & 0.0 & 39.0 \\
\hline \multicolumn{19}{|c|}{ Hole 67.1} \\
\hline Core & $\begin{array}{l}\text { Downhole } \\
\text { Depth of } \\
\text { Core }\end{array}$ & $\begin{array}{c}\text { Depth } \\
\text { Range } \\
\text { Sampled }\end{array}$ & Diff. & $\begin{array}{c}\% \\
\text { Amorphous }\end{array}$ & Quar. & Plag. & Kaol. & Mica & Mont. & Crist. & Clin. & $\mathrm{K}-\mathrm{Fe}$ & Pyrite & Barite & Apat. & Side. & Paly. & Phil. \\
\hline 1 & $23-32$ & $\begin{array}{l}23.65- \\
23.67\end{array}$ & 89.0 & 82.8 & 0.0 & 11.6 & 8.7 & 0.0 & 79.6 & 0.0 & 0.0 & 0.0 & 0.0 & 0.0 & 0.0 & 0.0 & 0.0 & 0.0 \\
\hline
\end{tabular}

${ }^{\text {a }}$ Abbreviations for X-ray mineralogy results: Quar.-quartz; Plag.-plagioclase; Kaol.-kaolinite; Mont.-montmorillonite; Crist.-cristobalite; Clin.-clinoptilolite; K-Fe-K-feldspar; Apat. -apatite; Side.-siderite; Paly.-palygorskite; Phil.-phillipsite.

* Depth on plot changed in order to accommodate samples received.

Note: Entries of zero indicate mineral not present in traceable quantity. 

PLATE 1

Palygorskite

Hole 66.0 , Core 8 , Section 5 
PLATE 1

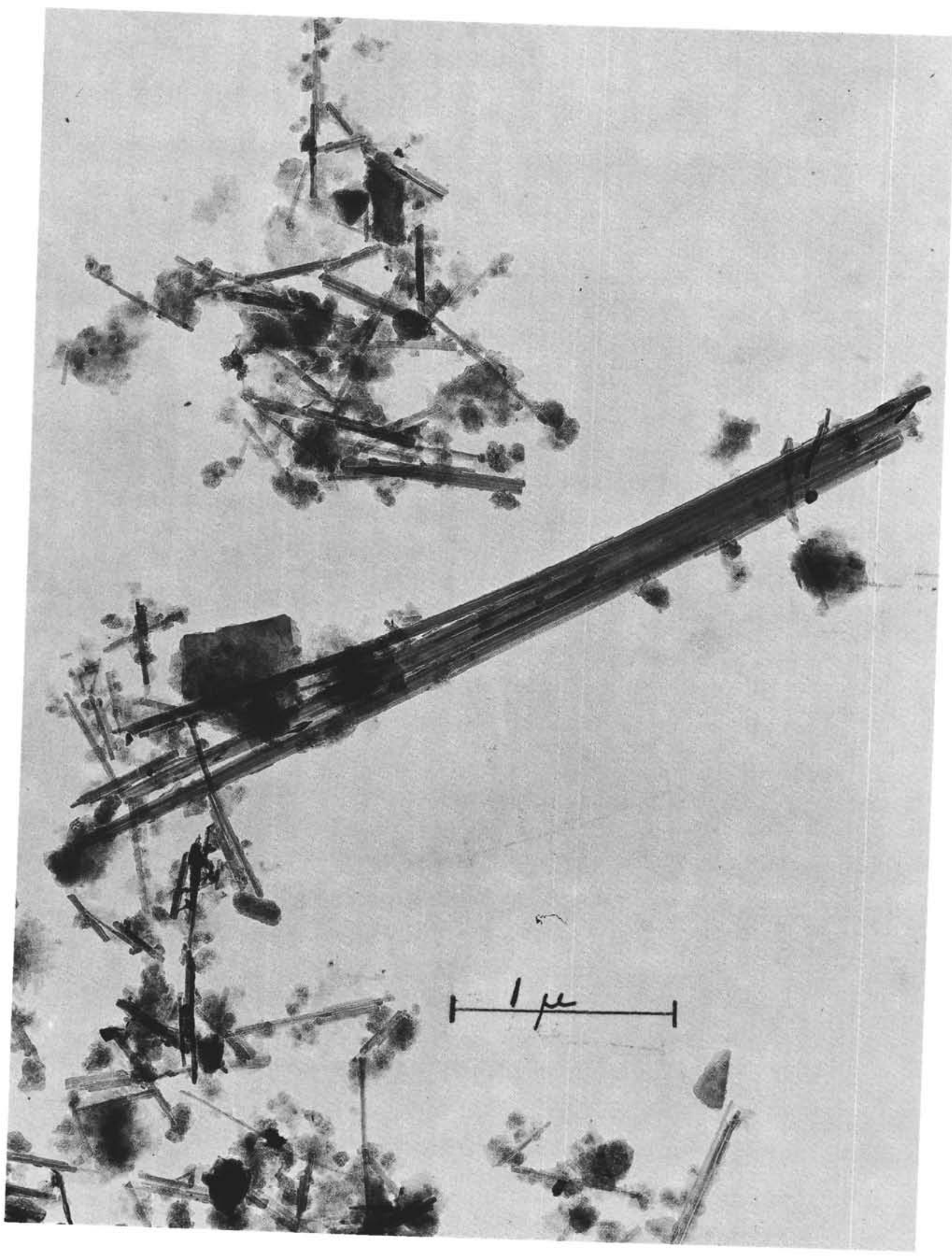

